\title{
D-mesons and charmonium states in hot isospin asymmetric strange hadronic matter
}

\author{
Arvind Kumar ${ }^{*}$ and Amruta Mishra ${ }^{\dagger}$ \\ Department of Physics, Indian Institute of Technology, Delhi, Hauz Khas, New Delhi - 110 016, India
}

\begin{abstract}
We study the properties of $D$ and $\bar{D}$ mesons in hot isospin asymmetric strange hadronic matter, arising due to their interactions with the hadrons in the hyperonic medium. The interactions of $D$ and $\bar{D}$ mesons with these light hadrons are derived by generalizing the chiral SU(3) model used for the study of hyperonic matter to $\mathrm{SU}(4)$. The nucleons, hyperons, the scalar isoscalar meson, $\sigma$ and the scalar-isovector meson, $\delta$ as modified in the strange hadronic matter, modify the masses of $D$ and $\bar{D}$ mesons. It is found that as compared to the $\bar{D}$ mesons $\left(\bar{D}^{0}, D^{-}\right)$, the properties of the $D$ mesons $\left(D^{0}, D^{+}\right)$are more sensitive to the isospin asymmetry at high densities. On the other hand, the effects of strangeness fraction are found to be more dominant for the $\bar{D}$ mesons as compared to $D$ mesons and these modifications are observed to be particularly appreciable at high densities. We also study the mass modifications of the charmonium states $J / \psi, \psi(3686)$ and $\psi(3770)$ in the isospin asymmetric strange hadronic matter at finite temperatures and investigate the possibility of the decay of the charmonium states into $D \bar{D}$ pairs in the hot hadronic medium. The mass modifications of these charmonium states arise due to their interaction with the gluon condensates of QCD, simulated by a scalar dilaton field introduced to incorporate the broken scale invariance of QCD within the effective chiral model. The effects of finite quark masses are taken into account in the trace of the energy momentum tensor in QCD, while investigating the medium modification of the charmonium masses through the modification of the gluon condensate in the medium. We also compute the partial decay widths of the charmonium states to the $D \bar{D}$ pairs in the hadronic medium. The strong dependence on density of the in-medium properties of the $D$, $\bar{D}$ and the charmonium states, as well as the partial decay widths of charmonium states to $D \bar{D}$ pairs, found in the present investigation, will be of direct relevance in observables like open charm enhancement as well as $J / \psi$ suppression in the compressed baryonic matter (CBM) experiments at the future Facility for Antiproton and Ion Research, GSI, where the baryonic matter at high densities is planned to be produced.
\end{abstract}

PACS numbers:

\section{INTRODUCTION}

The study of the medium modifications of hadrons is an important topic of research in strong interaction physics, which is of relevance in the heavy-ion collision experiments as well as in nuclear astrophysics. The hadrons e.g. the vector mesons, the kaons and the $D$ mesons are made up of a light quark $(\mathrm{u}, \mathrm{d})$ and/or a light antiquark $(\bar{u}, \bar{d})$. The mass of light quark is around $5-10 \mathrm{MeV}$ whereas the the mass of the hadron e.g. proton is about $939 \mathrm{MeV}$. The large mass of the hadron is attributed to the spontaneous breaking of the chiral symmetry of QCD, which leads to the population of the ground state of QCD with quark condensates. The masses of the hadrons are modified in the hadronic medium and these medium modifications can be linked with the medium modifications of the quark and/or gluon condensates of QCD. The modifications of the hadron properties are of direct relevance for the experimental observables of heavy ion collision experiments. The CERES experiment at CERN have observed an enhanced yield of dilepton pairs in heavy-ion reactions e.g. in S+Au collisions [1]. The HELIOS [2] and DLS [3] collaborations also report high yield of dielectrons in the nucleus-nucleus collisions. The above high observed yield of dileptons may be because of medium modifications of the vector mesons and their decay to dilepton pairs [4 [8]. The enhanced yield in dileptons can also be a signature of formation of thermalized quark gluon plasma [9]. The dilepton pairs and photons observed in the heavy-ion collisions are helpful in understanding the initial phase of collisions as dileptons interact in the medium only electromagnetically and most of the information of the collison zone remain undistorted [10, 11]. The properties of the kaons and antikaons in the hot and dense matter arising from heavy ion collision experiments have been studied by KaoS collaboration [12 15]. The observed production and collective flow indicate that the kaons

\footnotetext{
*Electronic address: iitd.arvind@gmail.com

${ }^{\dagger}$ Electronic address: amruta@physics.iitd.ac.in,mishra@th.physik.uni-frankfurt.de
} 
feel a repulsive interaction in the hadronic medium whereas antikaons undergo attractive interactions in the medium [5, 16 22]. The latter can lead to antikaon condensation in the neutron stars, as was suggested by Kaplan and Nelson [23].

The study of the mass modifications of $D$ mesons is relevant in understanding their production as well as collective flow in the heavy-ion collision experiments and have gotten considerable attention due to the experimental facilities, e.g., at Jefferson Lab, USA [24] and at the upcoming FAIR (Facility for Antiproton and Ion Research) project at GSI, Germany [25]. In Jefferson Lab, CEBAF (Continuous Electron Beam Acclerator Facility) acclerator is used to get the continuous beam of electrons and these are scattered off from nuclei to produce charm hadrons 24]. The CBM (Compressed Baryonic Matter) experiment at FAIR facility intends to explore the phase diagram of strongly interacting matter at high baryon densities and moderate temperatures in heavy ion collisions and the medium modification of the charm mesons are planned to be investigated in these experiments. The PANDA experiment of FAIR project, GSI, in the experiment with the annihilation of antiprotons on the nuclei, also intends to study $D$ meson and charmonium spectroscopy [26]. On the theoretical side, the properties of $D$ mesons have been investigated in quark meson coupling (QMC) model [27]. In quark meson coupling model, $D(\bar{D})$ mesons are assumed to be bound states of a light quark (antiquark) and a charm antiquark (quark), and they interact with the nucleons through the exchange of scalar and vector mesons. These studies suggest that the $D$ mesons undergo mass drop in the nuclear medium. The QCD sum rule (QSR) approach has also been used to study the medium modification of $D$ mesons in the nuclear medium [28 30]. In the QCD sum rule approach, the in-medium properties of the hadrons, are related to the QCD ground state properties e.g. the quark and/or gluon condensates. The $D(\bar{D})$ mesons, which are made up of one light antiquark (light quark), according to the QSR approach, undergo appreciable medium modification in their masses, because of their interaction with the light quark condensates in the nuclear medium. The mass drop of $D$ mesons observed in the QCD sum rule approach [28] turns out to be similar as in QMC model [27]. The coupled channel approach has also been vastly used in the literature to study the mass modifications of $D$ and $\bar{D}$ mesons in the nuclear medium at zero and finite temperatures [31 34]. The coupled channel approach has successfully been applied to understand the $\bar{K} N$ interactions in isospin I $=0$ channel and dynamics of resonance $\Lambda(1405)$ [35]. The interest in the study of charm mesons have been triggered by the discovery of the charmed baryonic resonances by CLEO, Belle and BABAR collaborations 36 [1]. However, it is a matter of interest to explore whether these resonances have $q q q$ structure or can be generated dynamically via meson baryon scattering processes 42]. The study of the properties of $D$ mesons for the first time was studied within the coupled channel approach in Ref. 31] by assuming a separable baryon meson potential and using the $\mathrm{SU}(3)$ symmetry in u,d and c quarks. In Ref. [31] the resonance $\Lambda_{c}(2593)$ was produced in isospin I $=0$ channel and the effects of the Pauli blocking of the nucleons, pion modification, baryon modification and self dressing of $D$ mesons were taken into account to investigate the medium modifications of the $D$ mesons. It was observed that there is only a small drop in the mass of $D$ mesons in the fully self-consistent calculations for the study of the $D$ mesons in the nuclear medium [31]. In Ref. [43], a coupled channel method was developed, which was based on $\mathrm{SU}(4)$ symmetry in $u, d, s$ and $c$ quarks. In this approach the free space scattering amplitudes were constructed through t-channel exchange of vector mesons and KSFR relations [44]. This method was later used to study the mass modifications of $D$ and $\bar{D}$ mesons in cold nuclear medium [45]. In Ref. [33, 34], the original model developed by Hoffmann and Lutz was modified in several aspects e.g. in terms of using cut-off regularization instead of dimensional regularization and was used to study the properties of $D$ mesons at zero [33] as well as at finite temperatures [34]. These studies generated a narrow resonance, $\Lambda_{c}(2593)$ in isospin $\mathrm{I}=0$ channel and a wide resonance, $\Sigma_{c}(2770)$ in $\mathrm{I}=1$ channel of $D N$ interactions. At zero temperature these resonances move to higher values of c.m. energies due to Pauli blocking effect, whereas the finite temperature effect leads to the movement of these resonances towards their free space position. The properties of $D$ and $D^{*}$ mesons have also been investigated using $\mathrm{SU}(8)$ spin flavor symmetry and lead to generation of rich spectrum of resonances, e.g., $\Lambda_{c}(2660), \Lambda_{c}(2941), \Sigma_{c}(2554), \Sigma_{c}(2902)$, with $J=3 / 2$ and $\Sigma_{c}(2770)$ and $\Sigma_{c}(2823)$ with $J=1 / 2$ [42, 46 48]. The mass modification of the $D$ meson in the nuclear medium may also affect the properties of scalar charm and hidden charm resonances. In Ref. [49], it is observed that the spectral widths of resonances $D_{s 0}(2317)$ and $X(3700)$ are modified considerably in the nuclear medium whereas the resonance $D(2400)$ is not affected much in the medium.

The present paper is devoted to the study of medium modifications of $D$ and $\bar{D}$ mesons in isospin asymmetric strange hadronic medium at finite temperatures. In the present work, we also investigate the properties of the charmonium states, $J / \psi, \psi(3686)$ and $\psi(3770)$ in isospin asymmetric strange hadronic matter at finite temperatures. The investigation of the mass modifications of $D$ mesons and charmonium states might be useful to understand anomalous $J / \psi$ suppression observed by NA50 collaboration in $\mathrm{Pb}-\mathrm{Pb}$ collisions [50 52]. The excited states of charmonium, considered as a major source of $J / \psi$ mesons [53] could decay to $D \bar{D}$ pairs [54] due to mass modifications of the $D$ and $\bar{D}$ mesons in the medium. The medium modifications of the $J / \psi$ mesons are observed to be small [55] as compared to the $D$ meson mass modifications [28, 56, 57]. According to QCD sum rules, this is attributed to be due to the reason 
that the $D$ mesons interact in the nuclear medium through light quark condensates, which undergo appreciable modification in the medium. On the other hand, within the QCD sum rule approach, the mass modifications of the $J / \psi$ mesons in the leading order are due to the medium modifications of the gluon condensates, and the gluon condensates have negligible modifications in the medium as compared to the medium modifications of the light quark condensates. The mass modification of the charmonium states in the medium have also been calculated from the self energy of $J / \psi$ due to the $D$ meson loop [55] as well as due to the $D^{*}$ meson loop [58]. The mass modification of the charmonium states due to the $D$-meson loop are observed to be smaller as compared to the effect due to the interaction with the gluon condensates, whereas, the mass modification of $J / \psi$ due to the $D^{*}$ meson loop is observed to be large [58]. In references [59] and [60], $J / \psi$ suppression can be due to the formation of QGP since the charmonium dissociation rate is larger in QGP phase as compared to in the hadronic medium. The additional $J / \psi$ suppression observed in SPS and RHIC energies can be due to co mover scattering [61 63]. The $J / \psi$ mesons can also undergo absorption in the hadronic medium 64 66. In the hadronic phase the $J / \psi$ mesons interact with the pions and $\rho$ mesons and form the charmed hadrons through reactions e.g. $\pi+J / \psi \rightarrow D^{*}+\bar{D}$ and therefore it is necessary to consider the medium modifications of mesons to explain the $J / \psi$ suppression phenomenon [61]. In Ref. [67] the phenomenon of $J / \psi$ suppression was studied at SPS energies in covariant transport approach, whereas in Ref. 64], the $J / \psi$ suppression was investigated at RHIC energies in $A u+A u$ and $S+S$ collisions. It was observed that for the heavy nuclei both the plasma screening and the gluon scattering are important and hadron absorption has only a minor effect, but for the light nuclei collisions the effect of the plasma screening is observed to be negligible [64].

In the present investigation, the isospin asymmetric hyperonic matter is described by a chiral SU(3) model [68]. The chiral $\mathrm{SU}(3)$ model is then generalized to SU(4) to study the in-medium properties of the $D$ and $\bar{D}$ mesons. However, since the chiral symmetry is broken for the SU(4) case by the large charm mass, we use the SU(4) symmetry only to derive the interactions of the $\mathrm{D}$ and $\bar{D}$ mesons in the hadronic medium, but use the observed values of the charmed hadron masses and empirical values of the decay constants [69]. In the present investigation, the medium modifications of the charmonium states are considered through the medium modification of a scalar dilaton field $\chi$ which is introduced in the effective chiral model to mimic the the trace anomaly of QCD and is related to the scalar gluon condensate of QCD [70] The chiral SU(3) model used in the present work has already been used to investigate the medium modifications of the vector mesons 71,72$]$ and the optical potentials of the kaons and antikaons [20 22]. The properties of the $D$ mesons in symmetric/asymmetric nuclear matter at zero and finite temperatures have also been studied [70, 73, 74] using the interactions of the charmed mesons, obtained by a generalization of the chiral $\mathrm{SU}(3)$ to $\mathrm{SU}(4)$. In the present investigation, we study the effects of the strangeness on the properties of the $D$ and $\bar{D}$ mesons. The properties of charmonium states in isospin asymmetric hot nuclear medium arising due to the medium modifications of the dilaton field using the effective chiral model have been investigated [70, 75] and the present work studies the effects of strangeness on the in-medium masses of the charmonium states.

The outline of the paper is as follows. In section 2, we give a brief introduction to the effective chiral $S U(3)$ model and its extension to SU(4) to study the medium modification of $D$ and $\bar{D}$ mesons in hot isospin asymmetric strange hadronic matter. In section 3, we write the interaction Lagrangian densities for $D$ and $\bar{D}$ mesons with baryons and scalar mesons and derive the dispersion relations for the $D$ and $\bar{D}$ mesons. These dispersion relations are then solved to obtain their optical potentials in the strange hadronic matter at finite temperatures. In section 4 , we investigate the in-medium masses of the charmonium states $J / \psi, \psi(3686)$ and $\psi(3770)$ arising from the medium modification of the scalar dilaton field, which is related to the modification of the gluon condensate in the hadronic medium. We also discuss the modifications of the size of the charmonium states in the hadronic medium. In section 5 , we present the expressions of the partial decay widths of the charmonium states to $D \bar{D}$ pairs, obtained within the $3 P 0$ model [57, 85, 86], and discuss our choice of parameters. In section 6 we discuss the results obtained for the medium modifications of the $D(\bar{D})$ mesons and the charmonium states in isospin asymmetric strange hadronic matter, as well as the effects of these modifications on the partial decay widths of the charmonium states to the $D \bar{D}$ pairs. One observes nodes in the decay widths at certain densities when one considers the mass modifications of the $(D \bar{D})$ mesons in the hadronic medium, as has been already observed in the literature [57]. However, appreciable modifications of these decay widths are found when the mass modifications of the charmonium states are also taken into account. Finally, in section 7 , we summarize the findings of the present investigation and discuss possible outlook.

\section{THE EFFECTIVE CHIRAL MODEL}

The chiral $S U(3)$ model [68] used in the present investigation for the study of the light hadrons is based on nonlinear realization of chiral symmetry [76 78] and broken scale invariance [68, 71, 72. A scalar dilaton field is introduced in the effective hadronic model to mimic the broken scale invariance of QCD [68, 70, 79]. 
The effective hadronic chiral Lagrangian contains the following terms

$$
\mathcal{L}=\mathcal{L}_{\text {kin }}+\sum_{W} \mathcal{L}_{B W}+\mathcal{L}_{\text {vec }}+\mathcal{L}_{0}+\mathcal{L}_{\text {scalebreak }}+\mathcal{L}_{S B}
$$

In Eq.(11), $\mathcal{L}_{k i n}$ is the kinetic energy term, $\mathcal{L}_{B W}$ is the baryon-meson interaction term of meson of type W, in which the baryons-spin-0 meson interaction term generates the baryon masses. $\mathcal{L}_{\text {vec }}$ describes the dynamical mass generation of the vector mesons via couplings to the scalar mesons and contains additionally quartic self-interactions of the vector fields. $\mathcal{L}_{0}$ contains the meson-meson interaction terms inducing the spontaneous breaking of chiral symmetry and the term $\mathcal{L}_{\text {scalebreak }}$ corresponds to the scale breaking potential [68, 70, 79] given as

$$
\mathcal{L}_{\text {scalebreak }}=-\frac{1}{4} \chi^{4} \ln \frac{\chi^{4}}{\chi_{0}^{4}}+\frac{d}{3} \chi^{4} \ln \left(\frac{\left(\sigma^{2}-\delta^{2}\right) \zeta}{\sigma_{0}^{2} \zeta_{0}}\left(\frac{\chi}{\chi_{0}}\right)^{3}\right)
$$

where $\chi, \sigma, \zeta$ and $\delta$ are the scalar dilaton field, non-strange scalar field, strange scalar field and the scalar-isovector field respectively. $\mathcal{L}_{S B}$ describes the explicit chiral symmetry breaking. To study the hadron properties at finite densities, we use the mean field approximation, where all the meson fields are treated as classical fields. In this approximation, only the scalar and the vector fields contribute to the baryon-meson interaction, $\mathcal{L}_{B W}$ since for all the other mesons, the expectation values are zero. The coupled equations of motion of the scalar fields $(\sigma, \zeta$ and $\delta)$ and the dilaton field, $\chi$, obtained from the mean field Lagrangian density, are solved to obtain their values in the isospin asymmetric strange hadronic medium at finite temperatures. These medium dependent scalar fields are then used to obtain the optical potentials for the $D$ and $\bar{D}$ mesons, as well as the in-medium masses of the charmonium states for given values of density, temperature, isospin asymmetry parameter, $\eta=-\frac{\sum_{i} I_{3 i} \rho_{i}}{\rho_{B}}$, with $\rho_{i}$ is the number density of the baryon of $i$-th type and the strangeness fraction, $f_{s}=\frac{\sum_{i} s_{i} \rho_{i}}{\rho_{B}}$, where $s_{i}$ is the number of strange quarks of baryon $i$. The same procedure was used to study the properties of the $D, \bar{D}$ mesons as well as charmonium states for the isospin asymmetric hot nuclear matter $\left(f_{s}=0\right)$ and the possibility of decay of the charmonium states to $D \bar{D}$ pairs in the hot nuclear medium was discussed in Ref. [70]. In the present work, we study also the effect of strangeness on the in-medium properties of these charmed mesons. As has already been mentioned, the medium modification of the masses of the charmonium states are investigated from the modification of the gluon condensates in the hadronic medium [55], which are related to the medium modification of the dilaton field in the effective chiral model used in the present investigation [70]. The comparison of the trace of the energy momentum tensor in QCD to the trace of the energy momentum tensor corresponding to the scale breaking term of the effective chiral model given by equation (22) leads to the relation of the scalar gluon condensate to the dilaton field as [75, 80],

$$
\theta_{\mu}^{\mu}=\left\langle\frac{\beta_{Q C D}}{2 g} G_{\mu \nu}^{a} G^{\mu \nu a}\right\rangle+\sum_{i} m_{i} \bar{q}_{i} q_{i} \equiv-(1-d) \chi^{4},
$$

where the second term in the trace accounts for the finite quark masses, with $m_{i}$ as the current quark mass for the quark of flavor, $i=u, d, s, c$. The one loop QCD $\beta$ function is given as

$$
\beta_{\mathrm{QCD}}(g)=-\frac{11 N_{c} g^{3}}{48 \pi^{2}}\left(1-\frac{2 N_{f}}{11 N_{c}}\right)
$$

where $N_{c}=3$ is the number of colors and $N_{f}$ is the number of quark flavors. Using equations (4) and (3), we obtain the scalar gluon condensate as related to the dilaton field, for $N_{f}=4$, as,

$$
\left\langle\frac{\alpha_{s}}{\pi} G_{\mu \nu}^{a} G^{\mu \nu a}\right\rangle=\frac{24}{25}\left[(1-d) \chi^{4}+\sum_{i} m_{i} \bar{q}_{i} q_{i}\right] .
$$

The second term, $\sum_{i} m_{i} \bar{q}_{i} q_{i}$ can be identified to be the negative of the explicit chiral symmetry breaking term $\mathcal{L}_{S B}$ of equation (11) [75] and is given as

$$
\sum_{i} m_{i} \bar{q}_{i} q_{i}=-\mathcal{L}_{S B}=\left[m_{\pi}^{2} f_{\pi} \sigma+\left(\sqrt{2} m_{k}^{2} f_{k}-\frac{1}{\sqrt{2}} m_{\pi}^{2} f_{\pi}\right) \zeta+\left(\sqrt{2} m_{D}^{2} f_{D}-\frac{1}{\sqrt{2}} m_{\pi}^{2} f_{\pi}\right) \zeta_{c}\right],
$$

where, $\zeta_{c}$ is the scalar charm quark condensate $\bar{c} c$. We thus see from the equation (5) that the scalar gluon condensate $\left\langle\frac{\alpha_{s}}{\pi} G_{\mu \nu}^{a} G^{\mu \nu a}\right\rangle$ is proportional to the fourth power of the dilaton field, $\chi$, in the limiting situation of massless quarks [70]. 


\section{III. $D$ AND $\bar{D}$ MESONS IN HOT ISOSPIN ASYMMETRIC STRANGE HADRONIC MATTER}

In this section we study the $D$ and $\bar{D}$ mesons properties in isospin asymmetric strange hadronic matter. As mentioned earlier, the medium modifications of the $D$ and $\bar{D}$ mesons arise due to their interactions with the nucleons, hyperons, and the scalar mesons.

The interaction Lagrangian density of the $D(\bar{D})$ meson is given as

$$
\begin{aligned}
\mathcal{L}_{i n t} & =-\frac{i}{8 f_{D}^{2}}\left[3\left(\bar{p} \gamma^{\mu} p+\bar{n} \gamma^{\mu} n\right)\left(\left(D^{0}\left(\partial_{\mu} \bar{D}^{0}\right)-\left(\partial_{\mu} D^{0}\right) \bar{D}^{0}\right)+\left(D^{+}\left(\partial_{\mu} D^{-}\right)-\left(\partial_{\mu} D^{+}\right) D^{-}\right)\right)\right. \\
& +\left(\bar{p} \gamma^{\mu} p-\bar{n} \gamma^{\mu} n\right)\left(\left(D^{0}\left(\partial_{\mu} \bar{D}^{0}\right)-\left(\partial_{\mu} D^{0}\right) \bar{D}^{0}\right)-\left(D^{+}\left(\partial_{\mu} D^{-}\right)-\left(\partial_{\mu} D^{+}\right) D^{-}\right)\right) \\
& +2\left(\left(\bar{\Lambda}^{0} \gamma^{\mu} \Lambda^{0}\right)\left(\left(D^{0}\left(\partial_{\mu} \bar{D}^{0}\right)-\left(\partial_{\mu} D^{0}\right) \bar{D}^{0}\right)+\left(D^{+}\left(\partial_{\mu} D^{-}\right)-\left(\partial_{\mu} D^{+}\right) D^{-}\right)\right)\right. \\
& +2\left(\left(\bar{\Sigma}^{+} \gamma^{\mu} \Sigma^{+}+\bar{\Sigma}^{-} \gamma^{\mu} \Sigma^{-}\right)\left(\left(D^{0}\left(\partial_{\mu} \bar{D}^{0}\right)-\left(\partial_{\mu} D^{0}\right) \bar{D}^{0}\right)+\left(D^{+}\left(\partial_{\mu} D^{-}\right)-\left(\partial_{\mu} D^{+}\right) D^{-}\right)\right)\right. \\
& \left.+\left(\bar{\Sigma}^{+} \gamma^{\mu} \Sigma^{+}-\bar{\Sigma}-\gamma^{\mu} \Sigma^{-}\right)\left(\left(D^{0}\left(\partial_{\mu} \bar{D}^{0}\right)-\left(\partial_{\mu} D^{0}\right) \bar{D}^{0}\right)-\left(D^{+}\left(\partial_{\mu} D^{-}\right)-\left(\partial_{\mu} D^{+}\right) D^{-}\right)\right)\right) \\
& +2\left(\bar{\Sigma}^{0} \gamma^{\mu} \Sigma^{0}\right)\left(\left(D^{0}\left(\partial_{\mu} \bar{D}^{0}\right)-\left(\partial_{\mu} D^{0}\right) \bar{D}^{0}\right)+\left(D^{+}\left(\partial_{\mu} D^{-}\right)-\left(\partial_{\mu} D^{+}\right) D^{-}\right)\right) \\
& +\left(\bar{\Xi}^{0} \gamma^{\mu} \Xi^{0}+\bar{\Xi}-\gamma^{\mu} \Xi^{-}\right)\left(\left(D^{0}\left(\partial_{\mu} \bar{D}^{0}\right)-\left(\partial_{\mu} D^{0}\right) \bar{D}^{0}\right)+\left(D^{+}\left(\partial_{\mu} D^{-}\right)-\left(\partial_{\mu} D^{+}\right) D^{-}\right)\right) \\
& \left.+\left(\bar{\Xi}^{0} \gamma^{\mu} \Xi^{0}-\bar{\Xi}^{-} \gamma^{\mu} \Xi^{-}\right)\left(\left(D^{0}\left(\partial_{\mu} \bar{D}^{0}\right)-\left(\partial_{\mu} D^{0}\right) \bar{D}^{0}\right)-\left(D^{+}\left(\partial_{\mu} D^{-}\right)-\left(\partial_{\mu} D^{+}\right) D^{-}\right)\right)\right] \\
& \left.+\frac{m_{D}^{2}}{2 f_{D}}\left[\left(\sigma+\sqrt{2} \zeta_{c}\right)\left(\bar{D}^{0} D^{0}+\left(D^{-} D^{+}\right)\right)+\delta\left(\bar{D}^{0} D^{0}\right)-\left(D^{-} D^{+}\right)\right)\right] \\
& -\frac{1}{f_{D}}\left[\left(\sigma+\sqrt{2} \zeta_{c}\right)\left(\left(\partial_{\mu} \bar{D}^{0}\right)\left(\partial^{\mu} D^{0}\right)+\left(\partial_{\mu} D^{-}\right)\left(\partial^{\mu} D^{+}\right)\right)+\delta\left(\left(\partial_{\mu} \bar{D}^{0}\right)\left(\partial^{\mu} D^{0}\right)-\left(\partial_{\mu} D^{-}\right)\left(\partial^{\mu} D^{+}\right)\right)\right] \\
& +\frac{d_{1}}{2 f_{D}^{2}}\left(\bar{p} p+\bar{n} n+\bar{\Lambda}^{0} \Lambda^{0}+\bar{\Sigma}^{+} \Sigma^{+}+\bar{\Sigma}^{0} \Sigma^{0}+\bar{\Sigma}^{-} \Sigma^{-}+\bar{\Xi}^{0} \Xi^{0}+\bar{\Xi}^{-} \Xi^{-}\right)\left(\left(\partial_{\mu} D^{-}\right)\left(\partial^{\mu} D^{+}\right)\right. \\
& \left.+\left(\bar{n} \partial_{\mu} \bar{D}^{0}\right)\left(\partial^{\mu} D^{0}\right)\right)+\frac{d_{2}}{2 f_{D}^{2}}\left[\left(\bar{p} p+\frac{1}{6} \bar{\Lambda}^{0} \Lambda^{0}+\bar{\Sigma}^{+} \Sigma^{+}+\frac{1}{2} \bar{\Sigma}^{0} \Sigma^{0}\right)\left(\partial_{\mu} \bar{D}^{0}\right)\left(\partial^{\mu} D^{0}\right)\right. \\
& \left.\left(\bar{\Lambda}^{0} \Lambda^{0}+\bar{\Sigma}^{-} \Sigma^{-}+\frac{1}{2} \bar{\Sigma}^{0} \Sigma^{0}\right)\left(\partial_{\mu} D^{-}\right)\left(\partial^{\mu} D^{+}\right)\right]
\end{aligned}
$$

In Eq. (7), the first term is the vectorial Weinberg Tomozawa interaction term, obtained from the baryonpseudoscalar meson interaction Lagrangian given for the SU(4) case as follows.

$$
\mathcal{L}_{\mathrm{WT}}=-\frac{1}{2} \sum_{i, j, k, l} \bar{B}_{i j k} \gamma^{\mu}\left(\left(\Gamma_{\mu}\right)_{l}{ }^{k} B^{i j l}+2\left(\Gamma_{\mu}\right)_{l}^{j} B^{i l k}\right),
$$

In the above, the baryons belong to the 20-plet representation and mesons belong to the 16-plet representation. The baryons are represented by the tensor $B^{i j k}$, which are antisymmetric in the first two indices [43]. The indices $i, j, k$ run from one to four, where one can read off the quark content of a baryon state by the identifications $1 \leftrightarrow u, 2 \leftrightarrow$ $d, 3 \leftrightarrow s, 4 \leftrightarrow c$. The baryon states are given as [43],

$$
\begin{aligned}
& B_{121}=p, \quad B_{122}=n, \quad B_{213}=\frac{2}{\sqrt{6}} \Lambda^{0}, \\
& B_{132}=\frac{1}{\sqrt{2}} \Sigma^{0}-\frac{1}{\sqrt{6}} \Lambda^{0}, \quad B_{231}=\frac{1}{\sqrt{2}} \Sigma^{0}+\frac{1}{\sqrt{6}} \Lambda^{0}, \\
& B_{232}=\Sigma^{-}, B_{311}=\Sigma^{+}, B_{233}=\Xi^{-}, B_{313}=\Xi^{0}
\end{aligned}
$$

where we have written down only the baryons containing the three light quarks, $\mathrm{u}, \mathrm{d}$ and s quarks. The second term in equation (7) is the scalar meson exchange term, which is obtained from the explicit symmetry breaking term

$$
\mathcal{L}_{S B}=-\frac{1}{2} \operatorname{Tr} A_{p}\left(u X u+u^{\dagger} X u^{\dagger}\right)
$$

where, $A_{p}$ given as,

$$
A_{p}=1 / \sqrt{2} \operatorname{diag}\left(m_{\pi}^{2} f_{\pi}, m_{\pi}^{2} f_{\pi}, 2 m_{K}^{2} f_{K}-m_{\pi}^{2} f_{\pi}, 2 m_{D}^{2} f_{D}-m_{\pi}^{2} f_{\pi}\right),
$$


$X$ is the scalar meson multiplet [74]. In the above, $u$ is given as,

$$
u=\exp \left[\frac{i}{\sqrt{2} \sigma_{0}} M \gamma_{5}\right]
$$

with $M=\frac{1}{\sqrt{2}} \pi_{a} \lambda_{a}$ as the pseudoscalar meson multiplet [74]. The next three terms of equation (77) $\left(\sim\left(\partial_{\mu} \bar{D}\right)\left(\partial^{\mu} D\right)\right)$ are the range terms. The first range term (with coefficient $\left(-\frac{1}{f_{D}}\right)$ ) is obtained from the kinetic energy term of the pseudoscalar mesons which is defined as,

$$
\mathcal{L}_{1 \text { strangeterm }}=\operatorname{Tr}\left(u_{\mu} X u^{\mu} X+X u_{\mu} u^{\mu} X\right) .
$$

where, $u_{\mu}$ is given as,

$$
u_{\mu}=-\frac{i}{2}\left[u^{\dagger}\left(\partial_{\mu} u\right)-u\left(\partial_{\mu} u^{\dagger}\right)\right]
$$

The range terms $d_{1}$ and $d_{2}$ of equation (7) are obtained from the expressions

$$
\mathcal{L}_{d_{1}}=\frac{d_{1}}{4} \sum_{i, j, k, l=1}^{4} \bar{B}_{i j k} B^{i j k}\left(u_{\mu}\right)_{l}^{m}\left(u^{\mu}\right)_{m}^{l}
$$

and

$$
\mathcal{L}_{d_{2}}=\frac{d_{2}}{2} \sum_{i, j, k, l=1}^{4} \bar{B}_{i j k}\left(u_{\mu}\right)_{l}{ }^{m}\left(u^{\mu}\right)_{m}{ }^{k} B^{i j l}
$$

respectively. We have not taken into account vector meson-pseudoscalar interactions in the present investigation as we have retained only the leading and next to leading order contributions and in the nonlinear realization of chiral symmetry, such a term arises as a higher order contribution [81].

The dispersion relations for the $D$ and $\bar{D}$ mesons are obtained by the Fourier transformations of equations of motion. These are given as

$$
-\omega^{2}+\vec{k}^{2}+m_{D}^{2}-\Pi(\omega,|\vec{k}|)=0
$$

where, $m_{D}$ is the vacuum mass of the $D(\bar{D})$ meson and $\Pi(\omega,|\vec{k}|)$ is the self-energy of the $D(\bar{D})$ mesons in the medium. The self-energy $\Pi(\omega,|\vec{k}|$,$) for the D$ meson doublet $\left(D^{0}, D^{+}\right)$arising from the interaction of Eq.(7) is given as

$$
\begin{aligned}
\Pi(\omega,|\vec{k}|) & =\frac{1}{4 f_{D}^{2}}\left[3\left(\rho_{p}+\rho_{n}\right) \pm\left(\rho_{p}-\rho_{n}\right)+2\left(\left(\rho_{\Sigma^{+}}+\rho_{\Sigma^{-}}\right) \pm\left(\rho_{\Sigma^{+}}-\rho_{\Sigma^{-}}\right)\right)\right. \\
& \left.+2\left(\rho_{\Lambda^{0}}+\rho_{\Sigma^{0}}\right)+\left(\left(\rho_{\Xi^{0}}+\rho_{\Xi^{-}}\right) \pm\left(\rho_{\Xi^{0}}-\rho_{\Xi^{-}}\right)\right)\right] \omega \\
& +\frac{m_{D}^{2}}{2 f_{D}}\left(\sigma^{\prime}+\sqrt{2} \zeta_{c}{ }^{\prime} \pm \delta^{\prime}\right)+\left[-\frac{1}{f_{D}}\left(\sigma^{\prime}+\sqrt{2}{\zeta_{c}}^{\prime} \pm \delta^{\prime}\right)+\frac{d_{1}}{2 f_{D}^{2}}\left(\rho_{p}^{s}+\rho_{n}^{s}\right.\right. \\
& \left.+\rho_{\Lambda^{0}}^{s}+\rho_{\Sigma^{+}}^{s}+\rho_{\Sigma^{0}}^{s}+\rho_{\Sigma^{-}}^{s}+\rho_{\Xi^{0}}^{s}+\rho_{\Xi^{-}}^{s}\right)+\frac{d_{2}}{4 f_{D}^{2}}\left(\left(\rho_{p}^{s}+\rho_{n}^{s}\right) \pm\left(\rho_{p}^{s}-\rho_{n}^{s}\right)+\frac{1}{3} \rho_{\Lambda^{0}}^{s}\right. \\
& \left.\left.+\left(\rho_{\Sigma^{+}}^{s}+\rho_{\Sigma^{-}}^{s}\right) \pm\left(\rho_{\Sigma^{+}}^{s}-\rho_{\Sigma^{-}}^{s}\right)+\rho_{\Sigma^{0}}^{s}\right)\right]\left(\omega^{2}-\vec{k}^{2}\right) .
\end{aligned}
$$

where the \pm signs refer to the $D^{0}$ and $D^{+}$mesons, respectively, and $\sigma^{\prime}\left(=\sigma-\sigma_{0}\right), \zeta_{c}^{\prime}\left(=\zeta_{c}-\zeta_{c 0}\right)$, and $\delta^{\prime}\left(=\delta-\delta_{0}\right)$ are the fluctuations of the scalar-isoscalar fields $\sigma, \zeta_{c}$ and the scalar-isoscalar field $\delta$ from their vacuum expectation values in the strange hyperonic medium. A non-zero value of the scalar isovector field, $\delta$ means the medium has isospin asymmetry and the vacuum expectation value of scalar isovector field $\delta$ will be zero. Also, the fluctuation, $\zeta_{c}^{\prime}$, in the heavy charm quark condensate $\left(\zeta_{c}=\bar{c} c\right)$ from the vacuum value has been observed to be negligible [82] and its 
contribution to the in-medium masses of $D$ and $\bar{D}$ mesons will be neglected in the present investigation. In equation (18), $\rho_{i}$ and $\rho_{i}^{s}$ are the number density and the scalar density of the baryon of type $i$ with $i=p, n, \Lambda, \Sigma^{ \pm, 0}, \Xi^{-, 0}$, and are defined as [70],

$$
\rho_{i}=\gamma_{i} \int \frac{d^{3} k}{(2 \pi)^{3}}\left(\frac{1}{e^{\left(E_{i}^{*}(k)-\mu_{i}^{*}\right) / T}+1}-\frac{1}{e^{\left(E_{i}^{*}(k)+\mu_{i}^{*}\right) / T}+1}\right)
$$

and

$$
\rho_{i}^{s}=\gamma_{i} \int \frac{d^{3} k}{(2 \pi)^{3}} \frac{m_{i}^{*}}{E_{i}^{*}(k)}\left(\frac{1}{e^{\left(E_{i}^{*}(k)-\mu_{i}^{*}\right) / T}+1}+\frac{1}{e^{\left(E_{i}^{*}(k)+\mu_{i}^{*}\right) / T}+1}\right)
$$

respectively, where $\gamma_{i}=2$ is the spin degeneracy factor.

For the $\bar{D}$ meson doublet $\left(\bar{D}^{0}, D^{-}\right)$, the expression for self-energy is given as,

$$
\begin{aligned}
\Pi(\omega,|\vec{k}|) & =-\frac{1}{4 f_{D}^{2}}\left[3\left(\rho_{p}+\rho_{n}\right) \pm\left(\rho_{p}-\rho_{n}\right)+2\left(\left(\rho_{\Sigma^{+}}+\rho_{\Sigma^{-}}\right) \pm\left(\rho_{\Sigma^{+}}-\rho_{\Sigma^{-}}\right)\right)\right. \\
& \left.+2\left(\rho_{\Lambda^{0}}+\rho_{\Sigma^{0}}\right)+\left(\left(\rho_{\Xi^{0}}+\rho_{\Xi^{-}}\right) \pm\left(\rho_{\Xi^{0}}-\rho_{\Xi^{-}}\right)\right)\right] \omega \\
& +\frac{m_{D}^{2}}{2 f_{D}}\left(\sigma^{\prime}+\sqrt{2} \zeta_{c}{ }^{\prime} \pm \delta^{\prime}\right)+\left[-\frac{1}{f_{D}}\left(\sigma^{\prime}+\sqrt{2} \zeta_{c}{ }^{\prime} \pm \delta^{\prime}\right)+\frac{d_{1}}{2 f_{D}^{2}}\left(\rho_{p}^{s}+\rho_{n}^{s}\right.\right. \\
& \left.+\rho_{\Lambda^{0}}^{s}+\rho_{\Sigma^{+}}^{s}+\rho_{\Sigma^{0}}^{s}+\rho_{\Sigma^{-}}^{s}+\rho_{\Xi^{0}}^{s}+\rho_{\Xi^{-}}^{s}\right)+\frac{d_{2}}{4 f_{D}^{2}}\left(\left(\rho_{p}^{s}+\rho_{n}^{s}\right) \pm\left(\rho_{p}^{s}-\rho_{n}^{s}\right)+\frac{1}{3} \rho_{\Lambda^{0}}^{s}\right. \\
& \left.\left.+\left(\rho_{\Sigma^{+}}^{s}+\rho_{\Sigma^{-}}^{s}\right) \pm\left(\rho_{\Sigma^{+}}^{s}-\rho_{\Sigma^{-}}^{s}\right)+\rho_{\Sigma^{0}}^{s}\right)\right]\left(\omega^{2}-\vec{k}^{2}\right),
\end{aligned}
$$

where the \pm signs refer to the $\bar{D}^{0}$ and $D^{-}$mesons, respectively. After finding the in-medium energies, $\omega(k)$ of $D$ and $\bar{D}$ mesons, we find their optical potentials using the expression

$$
U(k)=\omega(k)-\sqrt{k^{2}+m_{D}^{2}}
$$

where $m_{D}$ is the vacuum mass for the $D(\bar{D})$ meson.

\section{CHARMONIUM STATES IN HOT ASYMMETRIC STRANGE HADRONIC MATTER}

In this section, we outline the procedure which we use to study the medium modification of the masses as well as the sizes of the charmonium states $J / \psi, \psi(3686)$ and $\psi(3770)$ in hot isospin asymmetric strange hadronic matter. In the literature the masses of the charmonium states have been calculated using QCD sum rules through the medium modifications of the lowest dimension gluon condensate operators which consists of the scalar and twist-2 gluon condensates [83]. These operators can, in turn, be written in terms of the color electric field, $\left\langle\frac{\alpha_{s}}{\pi} \vec{E}^{2}\right\rangle$ and color magnetic field, $\left\langle\frac{\alpha_{s}}{\pi} \vec{B}^{2}\right\rangle$. However, in the non-relativistic limit, as the Wilson coefficients for the magnetic field part vanish, the lowest dimension gluon condensates can be written in terms of the electric field part only and the mass shift of the charmonium states can be calculated as a second order Stark effect in QCD, as has been studied in Ref. [55]. The expression for the mass shift of the charmonium state, derived in the large charm mass limit is given as [84]

$$
\Delta m_{\psi}=-\frac{1}{9} \int d k^{2}\left|\frac{\partial \psi(k)}{\partial k}\right|^{2} \frac{k}{k^{2} / m_{c}+\epsilon} \times\left(\left\langle\frac{\alpha_{s}}{\pi} E^{2}\right\rangle-\left\langle\frac{\alpha_{s}}{\pi} E^{2}\right\rangle_{0}\right) .
$$

In the above, $m_{c}$ is the mass of the charm quark, taken as $1.95 \mathrm{GeV}$ [55], $m_{\psi}$ is the vacuum mass of the charmonium state and $\epsilon=2 m_{c}-m_{\psi}$ is the binding energy. $\psi(k)$ is the wave function of the charmonium state in the momentum

space, normalized as $\int \frac{d^{3} k}{(2 \pi)^{3}}|\psi(k)|^{2}=1$ [89]. In the present investigation, the wave functions for the charmonium states are taken to be Gaussian and are given as [57]

$$
\psi_{N, l}(r)=\mathrm{N} \times Y_{l}^{m}(\theta, \phi)\left(\beta^{2} r^{2}\right)^{\frac{1}{2} l} \exp ^{-\frac{1}{2} \beta^{2} r^{2}} L_{N-1}^{l+\frac{1}{2}}\left(\beta^{2} r^{2}\right)
$$


where $N$ is the normalization constant, $\beta^{2}=M \omega / \hbar$ characterizes the strength of the harmonic potential, $M=m_{c} / 2$ is the reduced mass of the charm quark and charm anti-quark system, and $L_{p}^{k}(z)$ is the associated Laguerre Polynomial. As in Ref. [55], the oscillator constant $\beta$ is determined from the mean squared radii $\left\langle r^{2}\right\rangle$ as $0.47^{2} \mathrm{fm}^{2}, 0.96^{2} \mathrm{fm}^{2}$ and $1 \mathrm{fm}^{2}$ for the charmonium states $J / \psi, \psi(3686)$ and $\psi(3770)$, respectively. This gives the value for the parameter $\beta$ as $0.52 \mathrm{GeV}, 0.39 \mathrm{GeV}$ and $0.37 \mathrm{GeV}$ for $J / \psi, \psi(3686)$ and $\psi(3770)$, assuming that these charmonium states are in the $1 \mathrm{~S}, 2 \mathrm{~S}$ and $1 \mathrm{D}$ states respectively. [70],

In the non-relativistic limit the color electric field part can be written in terms of the scalar gluon condensate as

$$
\left\langle\frac{\alpha_{s}}{\pi} E^{2}\right\rangle=-\frac{1}{2}\left\langle\frac{\alpha_{s}}{\pi} G_{\mu \nu}^{a} G^{\mu \nu a}\right\rangle,
$$

which, in turn, gives the formula for the mass shift of the charmonium states as [70]

$$
\Delta m_{\psi}=\frac{1}{18} \int d k^{2}\left|\frac{\partial \psi(k)}{\partial k}\right|^{2} \frac{k}{k^{2} / m_{c}+\epsilon} \times\left(\left\langle\frac{\alpha_{s}}{\pi} G_{\mu \nu}^{a} G^{\mu \nu a}\right\rangle-\left\langle\frac{\alpha_{s}}{\pi} G_{\mu \nu}^{a} G^{\mu \nu a}\right\rangle_{0}\right) .
$$

The difference of the value of scalar gluon condensate in the medium and in the vacuum, using equations (5) and (6), can be written as,

$$
\begin{aligned}
& \left(\left\langle\frac{\alpha_{s}}{\pi} G_{\mu \nu}^{a} G^{\mu \nu a}\right\rangle-\left\langle\frac{\alpha_{s}}{\pi} G_{\mu \nu}^{a} G^{\mu \nu a}\right\rangle_{0}\right)=\frac{24}{25}\left[(1-d)\left(\chi^{4}-\chi_{0}^{4}\right)+m_{\pi}^{2} f_{\pi} \sigma^{\prime}\right. \\
+ & \left.\left(\sqrt{2} m_{k}^{2} f_{k}-\frac{1}{\sqrt{2}} m_{\pi}^{2} f_{\pi}\right) \zeta^{\prime}+\left(\sqrt{2} m_{D}^{2} f_{D}-\frac{1}{\sqrt{2}} m_{\pi}^{2} f_{\pi}\right) \zeta_{c}^{\prime}\right]
\end{aligned}
$$

where $\sigma^{\prime}, \zeta^{\prime}$ and $\zeta_{c}{ }^{\prime}$ are the fluctuations of the fields $\sigma, \zeta$ and $\zeta_{c}$ from their vacuum values. Using equation (27) in equation (26), we calculate the mass shifts for the charmonium states. As has already been mentioned in the last section, we neglect the fluctuation of the charm scalar field, $\zeta_{c}$, for the study of the in-medium properties of the $D$ and $\bar{D}$ mesons in the present investigation. We shall not take this into account also in the study of the in-medium masses of the charmonium states in the present study. We might note that when we do not consider the finite quark masses then the mass shift for the charmonium states is observed to be proportional to the shift in fourth power of the value of the dilaton field [70].

\section{DECAY WIDTHS OF THE CHARMONIUM STATES TO $D \bar{D}$ PAIRS}

In this section, we compute the partial decay widths of the charmonium states to $D \bar{D}$ pairs in the hot isospin asymmetric strange hadronic medium, by accounting for the internal structures of the parent and outgoing mesons using the 3P0 model [57, 85, 86]. The medium modifications of the masses of the $D(\bar{D})$ and the charmonium states as calculated in the present investigation, modify the decay widths of the charmonium states to $D \bar{D}$ pairs in the medium. The charmonium state at rest decays to the $D$ and $\bar{D}$ mesons and in the 3P0 model, this decay width is given as [86]

$$
\Gamma_{\psi \rightarrow D \bar{D}}=2 \pi \frac{p_{D} E_{D} E_{\bar{D}}}{M_{\psi}}|M|^{2}
$$

where, $M$ is the matrix element for the decay of the parent charmonium state to the $D \bar{D}$ pairs, $p_{D}$ is the magnitude of the 3-momentum of the $D(\bar{D})$ meson when the charmonium state $\psi$ decays at rest and is given by

$$
p_{D}=\left(\frac{M_{\psi}^{2}}{4}-\frac{m_{D}^{2}+m_{\bar{D}}^{2}}{2}+\frac{\left(m_{D}^{2}-m_{\bar{D}}^{2}\right)^{2}}{4 M_{\psi}^{2}}\right)^{1 / 2}
$$

and $E_{D}$ and $E_{\bar{D}}$ are the energies of the outgoing $D$ and $\bar{D}$ mesons given as $E_{D}=\left(p_{D}{ }^{2}+m_{D}{ }^{2}\right)^{1 / 2}$ and $E_{\bar{D}}=$ $\left(p_{D}{ }^{2}+m_{\bar{D}}^{2}\right)^{1 / 2}$, with $m_{D}$ and $m_{\bar{D}}$ as the masses of the $D$ and $\bar{D}$ mesons. In the isospin symmetric medium, the (almost) degeneracy in the masses of the $D^{+}$and $D^{0}$ mesons, as well as in the masses of the $D^{-}$and $\bar{D}^{0}$ mesons, leads to the partial decay widths of the charmonium states to $D^{+} D^{-}$pair and $D^{0} \bar{D}^{0}$ pairs as approximately equal. 
However, in the isospin asymmetric medium, the mass splitting between the masses of the $D^{+}$and $D^{0}$, as well as between the masses of the $D^{-}$and $\bar{D}^{0}$, lead to the partial decay widths for the channels when the charmonium state decays to $D^{+} D^{-}$and $D^{0} \bar{D}^{0}$ to be different. The decay widths for the charmonium states $J / \psi, \psi(3686)$ and $\psi(3770)$ decaying to $D \bar{D}\left(D^{+} D^{-}\right.$and $\left.D^{0} \bar{D}^{0}\right)$, are given as $[57]$

$$
\begin{gathered}
\Gamma(J / \psi \rightarrow D \bar{D})=\pi^{1 / 2} \frac{E_{D} E_{\bar{D}} \gamma^{2}}{2 M_{J / \psi}} \frac{2^{8} r^{3}\left(1+r^{2}\right)^{2}}{3\left(1+2 r^{2}\right)^{5}} x^{3} \times \exp \left(-\frac{x^{2}}{2\left(1+2 r^{2}\right)}\right), \\
\Gamma(\psi(3686) \rightarrow D \bar{D})=\pi^{1 / 2} \frac{E_{D} E_{\bar{D}} \gamma^{2}}{2 M_{\psi(3686)}} \times \frac{2^{7}\left(3+2 r^{2}\right)^{2}\left(1-3 r^{2}\right)^{2}}{3^{2}\left(1+2 r^{2}\right)^{7}} x^{2} \\
\times\left(1+\frac{2 r^{2}\left(1+r^{2}\right)}{\left(1+2 r^{2}\right)\left(3+2 r^{2}\right)\left(1-3 r^{2}\right)} x^{2}\right)^{2} \times \exp \left(-\frac{x^{2}}{2\left(1+2 r^{2}\right)}\right), \\
\Gamma(\psi(3770) \rightarrow D \bar{D})=\pi^{1 / 2} \frac{E_{D} E_{\bar{D}} \gamma^{2}}{2 M_{\psi(3770)}} \frac{2^{11} 5}{3^{2}}\left(\frac{r}{1+2 r^{2}}\right)^{7} \times x^{3}\left(1-\frac{1+r^{2}}{5\left(1+2 r^{2}\right)} x^{2}\right)^{2} \exp \left(-\frac{x^{2}}{2\left(1+2 r^{2}\right)}\right),
\end{gathered}
$$

In the above, $r=\frac{\beta}{\beta_{D}}$ is the ratio of the harmonic oscillator strengths of the decaying charmonium state and the produced $D(\bar{D})$-mesons, $x=p_{D} / \beta_{D}$ and $\gamma$ is a measure of the strength of the 3P0 vertex [57, 86] . Few comments regarding the above expressions for the decay widths may be in order. We might note here that the momentum dependence of the matrix element, $M$ of equation (28) for the decay of charmonium state to $D \bar{D}$ pair arises from an overlap integral of the wave function of the decaying charmonium with the same of the $D$ and $\bar{D}$ mesons within the 3P0 model [57, 87]. This overlap integral in general is a polynomial multiplied by a gaussian in the magnitude of 3-momentum, $p_{D}$ of the produced $D(\bar{D})$ in the center of mass frame. Therefore, the nodes of the wave functions in the coordinate space can lead to nodes in the momentum, $p_{D}$ for the decay amplitude. In particular, the polynomial part in equation (31) arises from the radial part of the corresponding $2^{3} S_{1}$ wave function of $\psi(3686)$ charmonium state, while the same in equation (32) arises from the orbital angular momentum part of the wave function of $1^{3} D_{1}$ wave function of $\psi(3770)$ state [57]. Since the magnitude of the $D(\bar{D}), p_{D}$ depends only upon the masses of the charmonium and the $D(\bar{D})$ mesons, as can be seen from equation (29), the medium modifications of the masses of these mesons can lead to vanishing of the decay widths at finite densities and temperatures. Such suppression of the decay widths arising from the internal structure of the mesons has already been observed for strong decays of charmonium in the vacuum [87] as well as in the medium [57]. In the present study, we also observe such a suppression of the decay widths for the decay of the charmonium states in the isospin asymmetric hot strange hadronic matter, as shall be discussed in the following section.

As has already been stated in the previous section, the strength of the harmonic oscillator potential for the charmonium state, $\beta$ is taken to be $0.52 \mathrm{GeV}, 0.39 \mathrm{GeV}$ and $0.37 \mathrm{GeV}$ for $J / \psi, \psi(3686)$ and $\psi(3770)$ as fitted from their a root mean squared radii, $\left\langle r^{2}\right\rangle$ as $0.47^{2} \mathrm{fm}^{2}, 0.96^{2} \mathrm{fm}^{2}$ and $1 \mathrm{fm}^{2}$, respectively [55]. The values of $\beta_{D}$ and $\gamma$ are taken to be $0.31 \mathrm{GeV}$ and 0.35 respectively [55], so as to reproduce the decay width of $\psi(3770)$ to $D \bar{D}$ and partial decay widths of $\psi(4040)$ to $D \bar{D}, D \bar{D}^{*}, \bar{D} D^{*}$ and $D^{*} \bar{D}^{*}$ in vacuum [57, 87]. With the values chosen for ratio of the strengths of the charmonium states and $D(\bar{D})$ mesons and the 3P0 vertex strength, $\gamma$, the partial decay width of $\psi(3770)$ to $D \bar{D}$ turns out to be $28.65 \mathrm{MeV}$, with the partial decay widths for the subchannels of $\psi(3770) \rightarrow D^{+} D^{-}$ and $\psi(3770) \rightarrow D^{0} \bar{D}^{0}$ as $12.16 \mathrm{MeV}$ and $16.49 \mathrm{MeV}$ respectively [88]. With the strength of the outgoing $D$ and $\bar{D}$ mesons taken as $\beta_{D}=0.31 \mathrm{GeV}$, the value of $r=\frac{\beta}{\beta_{D}}$ turns out to be $1.68,1.26$ and 1.19 for $J / \psi, \psi(3686)$ and $\psi(3770)$ respectively [55] in the present investigation. It may be noted here that in Ref. [57], the decay widths of the charmonium states into $D \bar{D}$ pairs in isospin symmetric hadronic matter were computed within the $3 \mathrm{P} 0$ model, using the ratio of the strengths of the charmonium to $D(\bar{D})$ to be $r=1.04$ for all the charmonium states. The expressions for the partial decay widths for the charmonium states to the $D \bar{D}$ pairs as given by the equations (30), (31) and (32) account for the fact that the $D$ and $\bar{D}$ mesons can have different masses in the hadronic medium and these masses have been calculated in the strange hadronic medium in the present investigation. We might note here that the earlier calculation of the decay widths of the charmonium states to $D \bar{D}$ pairs [57] had assumed the masses of the $D$ and $\bar{D}$ to be the same in the medium. The partial decays widths are computed in the isospin asymmetric strange hadronic medium, accounting for the medium modifications of the charmonium states and the $D$ and $\bar{D}$ mesons, as calculated in the present investigation. The results for the medium modifications of these states as well as their effects on the partial decay widths of the charmonium states to $D \bar{D}$ pairs are discussed in the next section. 


\section{RESULTS AND DISCUSSIONS}

In this section, we present the numerical results of our investigation of the in-medium properties of $D$ and $\bar{D}$ mesons and of the charmonium states in isospin asymmetric strange hadronic matter at finite temperatures, as well as the effects of these modifications on the decay of the charmonium states to the $D \bar{D}$ pairs in the hadronic medium. The decay widths of the charmonium states to the $D \bar{D}$ pairs have also been studied using the mass modifications of the charmonium states and $D(\bar{D})$ mesons calculated within the present model as well as accounting for the internal structure of these mesons using the 3P0 model. Within the parameter set used in the chiral effective model [0], the vacuum values of the non strange scalar isoscalar fields, $\sigma$ and strange scalar isoscalar field, $\zeta$ and the dilaton field $\chi$ are $-93.3 \mathrm{MeV},-106.6 \mathrm{MeV}$ and $409.8 \mathrm{MeV}$ respectively. The values, $g_{\sigma N}=10.6$ and $g_{\zeta N}=-0.47$ are determined by fitting vacuum baryon masses. The other parameters fitted to the asymmetric nuclear matter saturation properties in the mean-field approximation are: $g_{\omega N}=13.3, g_{\rho p}=5.5, g_{4}=79.7, g_{\delta p}=2.5, m_{\zeta}=1024.5 \mathrm{MeV}, m_{\sigma}=466.5$ $\mathrm{MeV}$ and $m_{\delta}=899.5 \mathrm{MeV}$. The values of the couplings of hyperons with the scalar fields are: $g_{\sigma \Lambda}=7.52, g_{\zeta \Lambda}=5.8$, $g_{\delta \Lambda}=0, g_{\sigma \Sigma}=6.13, g_{\zeta \Sigma}=5.8, g_{\delta \Sigma^{+}}=6.79, g_{\delta \Sigma^{-}}=-6.79, g_{\delta \Sigma^{0}}=0, g_{\sigma \Xi}=3.78, g_{\zeta \Xi}=9.14, g_{\delta \Xi^{0}}=2.36$ and $g_{\delta \Xi^{-}}=-2.36$. The values of couplings of hyperons with the vector mesons are: $g_{\omega \Lambda}=g_{\omega \Sigma}=\frac{2}{3} g_{\omega N}, g_{\rho \Sigma^{+}}=$ $\frac{2}{3} g_{\omega N}, g_{\rho \Sigma^{-}}=-\frac{2}{3} g_{\omega N}, g_{\rho \Sigma^{0}}=0, g_{\omega \Xi}=\frac{1}{3} g_{\omega N}, g_{\rho \Lambda}=0, g_{\rho \Xi^{0}}=\frac{1}{3} g_{\omega N}, g_{\rho \Xi^{-}}=-\frac{1}{3} g_{\omega N} g_{\phi \Lambda}=g_{\phi \Sigma}=-\frac{\sqrt{2}}{3} g_{\omega N}, g_{\phi \Xi}$ $=-\frac{2 \sqrt{2}}{3} g_{\omega N}\left[68\right.$. The coefficients $d_{1}$ and $d_{2}$ of the range term interactions of the $D$ and $\bar{D}$ mesons are calculated from the empirical values of the $K N$ scattering lengths for $I=0$ and $I=1$ channels, and are given as $2.56 / m_{K}$ and $0.73 / m_{K}$, respectively 22,70$]$.

\section{A. $D(\bar{D})$ mesons in the hadronic matter}

The $D$ and $\bar{D}$ mesons properties in hot isospin asymmetric strange hadronic matter are modified due to their interactions with nucleons, hyperons, the scalar $\sigma$ meson, and scalar isovector $\delta$ meson. As mentioned earlier, the non-strange scalar meson $\sigma$, the strange scalar meson $\zeta$, the scalar isovector meson $\delta$ and the dilaton field, $\chi$ are modified in the hadronic medium and their values are obtained by solving the coupled equations of motion of these scalar fields for given values of density, temperature, isospin asymmetry parameter and strangeness fraction of the hot hyperonic matter. The behavior of these scalar fields in isospin asymmetric nuclear medium $\left(f_{s}=0\right)$ at zero as well as finite temperatures have been studied in detail in Ref. 70, 74]. In the present work, we shall focus on the effect of the strangeness fraction of the medium on these scalar fields. For $\mathrm{T}=0$, density $\rho_{B}$ and a given isospin asymmetry $\eta$, the magnitude of the scalar field $\sigma$ is observed to increase with increase in the strangeness fraction in the medium at small densities, whereas at higher densities $\left(\rho_{B}>3.5 \rho_{0}\right)$, the magnitude of $\sigma$ is seen drop with increase in $f_{s}$. On the other hand, the magnitude of $\zeta$ is observed to decrease with the strangeness in the medium. It is found that in isospin asymmetric strange medium the magnitude of the scalar isovector field $\delta$ increases as we move from non strange medium $\left(f_{s}=0\right)$ to strange medium (finite $f_{s}$ ). However, at $\eta=0$, the value of $\delta$ is zero because of isospin symmetry of the hadronic medium as expected, since $\delta$ attains nonzero values only for the isospin asymmetric hadronic matter. At given values for temperature, density and $\eta$, the value of the dilaton field is observed to decrease with the increase in the strangeness fraction of the medium.

The effects of individual terms of Lagrangian density, given by equation (77), on the energies of $D$ and $\bar{D}$ mesons in isospin asymmetric hot nuclear matter have been studied in Refs. [70, 74]. Here, we shall discuss the effect of strangeness fraction, $f_{s}$ of the medium on the energies of $D$ and $\bar{D}$ mesons arising due to the various terms of the Lagrangian density given by equation (77). For a given value of density, $\rho_{B}$ and isospin asymmetry parameter, $\eta$, as we move from nuclear medium $\left(f_{s}=0\right)$ to the hyperonic matter $\left(f_{s} \neq 0\right)$, the attractive (repulsive) contribution to the in-medium energies of the $D(\bar{D})$ mesons from the Weinberg-Tomozawa term is observed to become smaller. The scalar meson exchange term is attractive for both the $D$ and $\bar{D}$ mesons. The increase in the magnitude of the $\sigma$ field with strangeness at low densities, corresponds to a smaller value for $\sigma^{\prime}$, the fluctuation of $\sigma,\left(\sigma^{\prime}=\right.$ sigma $\left.-\sigma_{0}\right)$, leading to a smaller drop in the masses of the $D$ and $\bar{D}$ mesons as compared to their masses in nuclear matter $\left(f_{s}=0\right)$. On the other hand, at high densities, there is a further drop of the $D$ and $\bar{D}$ meson masses when the strangeness fraction of the hadronic matter is increased. Due to the behavior of the $\sigma$ field with strangeness as described above, the magnitude of the first range term drops at small densities, whereas this term leads to an increase in the masses of the $\mathrm{D}$ and $\bar{D}$ mesons at high baryon densities, as we move from the nuclear medium $\left(f_{s}=0\right)$ to hyperonic matter (nonzero $f_{s}$ ). Also, as we increase the strangeness fraction of the medium, the $d_{1}$ term is observed to become more attractive whereas the $d_{2}$ term becomes less attractive. The attractive nature of $d_{1}$ term is found to be dominating over the first range term and the $d_{2}$ term. Due to this reason, the contribution of the total range term on the $\mathrm{D}$ and 
$\bar{D}$ masses is found to be more attractive with the increase in the strangeness fraction of the medium.

In Fig. 1, we show the variation of energies of $D^{+}[\operatorname{subplots}(\mathrm{a}),(\mathrm{c}),(\mathrm{e})]$ and $D^{0}[$ subplots (b), (d), (f)] mesons, at zero momentum, with the baryon density, for temperatures $\mathrm{T}=0,100$ and $150 \mathrm{MeV}$ for different values of the strangeness fraction. In each subplot we show the results for isospin asymmetric medium with $\eta=0.5$ and compare these energies with the values for symmetric hyperonic medium. In isospin symmetric strange hadronic medium, for a given value of density, the energies of $D^{+}$and $D^{0}$ mesons are observed to drop with increase in the strangeness fraction, $f_{s}$, of the medium and this drop is seen to be larger at higher densities. For example, at nuclear saturation density $\rho_{0}$ and for the isospin asymmetry parameter $\eta=0$, as we move from $f_{s}=0$ to 0.5 , the energies of both $D^{+}$and $D^{0}$ mesons are observed to decrease by about $1 \mathrm{MeV}$, whereas for $\rho_{B}=4 \rho_{0}$, there is seen to be a drop of about $31 \mathrm{MeV}$ from the value of $1524 \mathrm{MeV}(1520 \mathrm{MeV})$ for $D^{+}\left(D^{0}\right)$ meson, as $f_{s}$ is changed from 0 to 0.5 . In isospin asymmetric matter, the drop in the $\mathrm{D}$ meson masses with $f_{s}$ is seen to be much larger than for symmetric matter. For example, for $\eta=0.5$, at $4 \rho_{0}$ the energy of $D^{+}$is observed to decrease by about $68 \mathrm{MeV}$ for $f_{s}=0.5$, from the value from $1487 \mathrm{MeV}$ at $f_{s}=0$. The energy of $D^{0}$ meson in isospin asymmetric strange hadronic medium $(\eta=0.5)$ is observed to decrease by about $26(109) \mathrm{MeV}$ from the value of $1808(1842) \mathrm{MeV}$ at baryon density of $\rho_{0}\left(4 \rho_{0}\right)$ as we move from $f_{s}=0$ to 0.5 .

For given values of isospin asymmetry and strangeness fraction, the energies of the $D$ mesons $\left(D^{0}, D^{+}\right)$are found to drop with increase in the density of the medium. In isospin symmetric nuclear medium $(\eta=0)$ at zero temperature, the energy of $D^{+}\left(D^{0}\right)$ meson at zero momentum is observed to decrease by about 77.3 (77.2) MeV and 345 (344) $\mathrm{MeV}$ from its vacuum value, at $\rho_{0}$ and $4 \rho_{0}$ respectively. In isospin symmetric strange hadronic medium $(\eta=0)$, at the value of the strangeness fraction $f_{s}=0.5$, the energy of $D^{+}\left(D^{0}\right)$ meson at $|\vec{k}|=0$ decreases by 78.3(78.1) and $376.2(375.5) \mathrm{MeV}$ at $\rho_{0}$ and $4 \rho_{0}$ respectively from its vacuum value. The large drop in the masses of $D$ mesons in the hyperonic medium is because of decrease in the mass of $D$ mesons with strangeness fraction of the medium, as has already been discussed. In isospin asymmetric strange hadronic medium $\left(\eta=0.5, f_{s}=0.5\right)$, the energy of $D^{+}\left(D^{0}\right)$ is observed to decrease drop $92(82) \mathrm{MeV}$ and 450 (362) $\mathrm{MeV}$ at densities of $\rho_{0}$ and $4 \rho_{0}$ respectively from its vacuum value.

The results stated above for $D$ mesons are for the strange hadronic medium at zero temperature. As we move to the finite temperature of the strange hadronic medium the masses of the $D$ mesons increase and hence they have smaller drop from the vacuum values as compared to the zero temperature case. This has also been observed in nuclear medium calculation in Ref. 70]. In isospin symmetric strange hadronic medium with strangeness fraction $f_{s}=0.5$ and at a baryon density of $\rho_{0}\left(4 \rho_{0}\right)$ the mass of $D^{+}$meson is observed to drop by 72(357), 66(329) and 63(313) $\mathrm{MeV}$ from its vacuum value at temperatures $\mathrm{T}=50,100$ and $150 \mathrm{MeV}$ respectively. In isospin asymmetric medium $(\eta=0.5)$ medium, at strangeness fraction, $f_{s}=0.5$, and at a baryon density of $\rho_{0}\left(4 \rho_{0}\right)$ the energy of $D^{+}$meson is seen to decrease by $85(429), 79(390)$ and $78.6(374) \mathrm{MeV}$ at temperatures $\mathrm{T}=50,100$ and $150 \mathrm{MeV}$ respectively. For $f_{s}=0.5$ and $\eta=0.5$, at a density of $\rho_{0}\left(4 \rho_{0}\right)$, the values of mass drop for $D^{0}$ mesons are observed to be $75(341), 66(300)$ and $60(286) \mathrm{MeV}$ at temperatures $\mathrm{T}=50,100$ and $150 \mathrm{MeV}$ respectively.

In Fig. 2. we show the variation of the energies of $D^{-}$[subplots (a), (c), (e)] and $\overline{D^{0}}$ [subplots (b), (d), (f)] mesons, at zero momentum, with density, for temperatures $\mathrm{T}=0,100$ and $150 \mathrm{MeV}$. In each subplot, we show the results for given values of the strangeness fraction, for the isospin asymmetric strange hadronic medium $(\eta=0.5)$ and compared with the results for the symmetric matter $(\eta=0)$. At temperature $\mathrm{T}=0$, in isospin symmetric medium $(\eta=0)$, for a given value of density, as we move from $f_{s}=0$ to $f_{s}=0.5$, the energy of $D^{-}$meson is observed to decrease by $10(71) \mathrm{MeV}$ from the value of $1842(1708) \mathrm{MeV}$ at $f_{s}=0$, at a density of $\rho_{0}\left(4 \rho_{0}\right)$. The decrease in the energy of $\bar{D}$ mesons as a function of the strangeness fraction of the medium is because of the drop in the mass with strangeness due to the Weinberg Tomozawa term as well as the total range term. In the total range term, the attractive $d_{1}$ term dominates over the other range terms, as has already been discussed earlier. For the value of the isospin asymmetry parameter $\eta=0.5$, the energy of $D^{-}$meson decreases by 7 and $118 \mathrm{MeV}$ from the values of 1843 and $1732 \mathrm{MeV}$ at densities of $\rho_{0}$ and $4 \rho_{0}$ respectively when we increase the value of strangeness $f_{0}$ from 0 to 0.5 . In isospin symmetric medium $(\eta=0)$, as we move from $f_{s}=0$ to 0.5 , the energy of $\bar{D}^{0}$ meson is seen to decrease by about $10 \mathrm{MeV}$ and $71 \mathrm{MeV}$ from the values of 1838 and $1704.5 \mathrm{MeV}$ at $\rho_{0}$ and $4 \rho_{0}$ respectively. At $\eta=0.5$, the energy of $\bar{D}^{0}$ meson is seen to decrease by 35 (156) MeV from the value of $1842(1739) \mathrm{MeV}$ at a baryon density of $\rho_{0}\left(4 \rho_{0}\right)$. As has already been mentioned, we thus observe in the present investigation, the masses of the $\bar{D}$ mesons are more sensitive to the strangeness of the medium as compared to the masses of D mesons, particularly at high densities.

Now we shall discuss the behavior of the $\bar{D}$ mesons in the strange hadronic medium at finite temperatures. At finite temperatures, the drop in these masses are observed to be smaller than that of the zero temperature case. In isospin symmetric medium $(\eta=0)$, at baryon density $\rho_{B}=\rho_{0}$ and strangeness fractions $f_{s}=0(0.5)$, the mass of $D^{-}$ meson is seen to decrease by $20(30), 14(24)$ and $10(21) \mathrm{MeV}$ from its vacuum value at values of temperature, $\mathrm{T}=50$, 

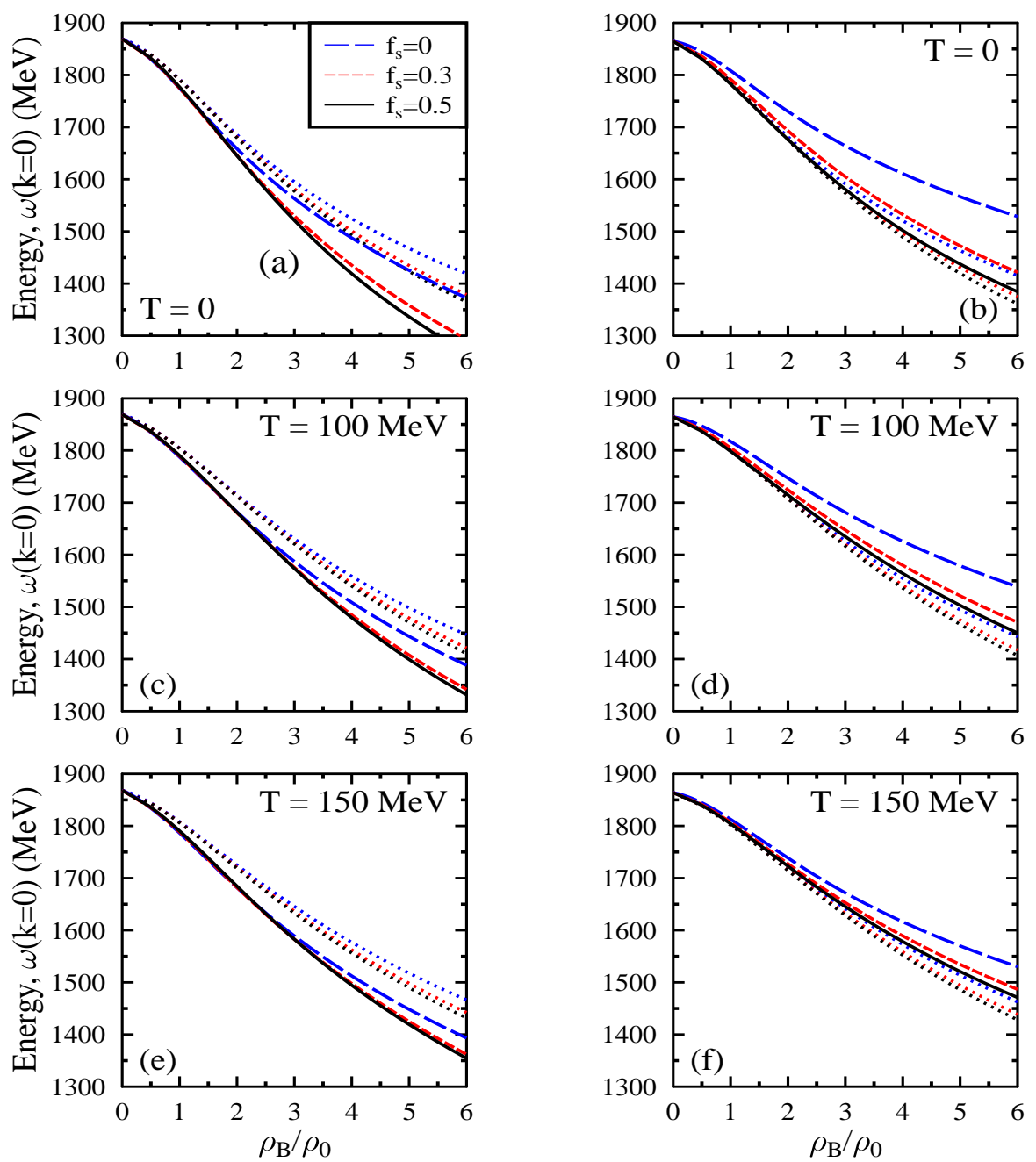

FIG. 1: (Color online) The energies of $D^{+}$(subplots a, c, e) and $D^{0}$ (subplots b, d, f) mesons at zero momentum in isospin asymmetric $(\eta=0.5)$ hyperonic medium for different temperatures, plotted as functions of baryon density in units of nuclear matter saturation density, $\rho_{B} / \rho_{0}$, for various values of the strangeness fraction, $f_{s}$. The results are compared with the case of isospin symmetric matter $(\eta=0)$ shown as dotted lines.

100 and $150 \mathrm{MeV}$ respectively, which may be compared to the zero temperature value of 27 (36.5) MeV for the mass drop. For baryon density $\rho_{B}=4 \rho_{0}$, for $\eta=0$ and $f_{s}=0(0.5)$, the values of the mass drop for $D^{-}$mesons are modified to $147(210), 121(178)$ and $100(160) \mathrm{MeV}$ at temperatures $\mathrm{T}=50,100$ and $150 \mathrm{MeV}$ respectively, which are smaller than the zero temperature values of mass drop of $161.5(228.5) \mathrm{MeV}$. In isospin asymmetric medium $(\eta=0.5)$, at baryon density $\rho_{B}=\rho_{0}$ and for the value of the strangeness fraction $f_{s}=0(0.5)$, the mass of $D^{-}$meson decreases by $20.5(26), 14(20)$ and $15(19.7) \mathrm{MeV}$ at the value of the temperature $\mathrm{T}=50,100$ and $150 \mathrm{MeV}$ respectively, whereas at baryon density $\rho_{B}=4 \rho_{0}$ these values of mass drop change to 128(229), 111(182) and 107(163) MeV respectively. For $\bar{D}^{0}$ mesons, in isospin asymmetric medium $(\eta=0.5)$, at baryon density, $\rho_{0}$ and for the strangeness fraction $f_{s}=0(0.5)$ the mass decreases by $18(51), 13(42)$ and $18(36) \mathrm{MeV}$ at the values of the temperature, $\mathrm{T}=50,100$ and $150 \mathrm{MeV}$ 
respectively from the vacuum value. For the same values for the isospin asymmetry and strangeness fraction, the values of the mass drop changes to $119(259), 110(214)$ and $120(198) \mathrm{MeV}$, at baryon density, $\rho_{B}=4 \rho_{0}$, at the value of the temperature, $\mathrm{T}=50,100$ and $150 \mathrm{MeV}$ respectively.

In the present investigation, we observe that the effect of strangeness fraction of the medium is to decrease the energies of the $D$ and $\bar{D}$ mesons. However, the $\bar{D}$ mesons are observed to undergo larger drop in their masses with increase in the strangeness fraction of the medium as compared to the masses of the D mesons. The difference in energies of $D$ and $\bar{D}$ mesons is due to the opposite sign in the contribution from the Weinberg Tomozawa term. With inclusion of hyperons in the medium, this term becomes less repulsive (attractive) for $\bar{D}$ (D) mesons leading to an decrease (increase) in the energy of $\bar{D}(\mathrm{D})$ mesons arising due to this term, as we increase $f_{s}$. This observed behavior of the contribution to the energy of $\mathrm{D}(\bar{D})$ mesons arising from the Weinberg-Tomozawa term can be understood in the following manner. For example, for a given density, for isospin symmetric nuclear matter, the expression in the bracket in the Weinberg-Tomozawa term in the self-energies of the $\mathrm{D}$ and $\bar{D}$ mesons given by equations (18) and (21) becomes equal to $3 \rho_{B}$. However, in the presence of hyperons, but in isospin symmetric matter, the expression in the bracket in this term becomes equal to $\left[3 \rho_{B}-\left(\rho_{\Lambda}+\rho_{\Sigma^{+}}+\rho_{\Sigma^{-}}+\rho_{\Sigma^{0}}+\rho_{\Xi^{-}}+\rho_{\Xi^{0}}\right)\right]$, which is less than $3 \rho_{B}$. Hence, for a given baryon density, the magnitude of the Weinberg-Tomozawa term is observed to decrease with increase in the strangeness fraction in the medium. The contributions to both the $\mathrm{D}$ and $\bar{D}$ mesons from the scalar exchange term as well as the range terms are the same. The scalar meson exchange term is seen to decrease the energies of $D$ and $\bar{D}$ mesons, with increase in the strangeness in the medium, at densities up to about $3.5 \rho_{0}$ and leads to an increase at higher densities. However, the modification in the energies of the $\mathrm{D}$ and $\bar{D}$ mesons due to increase in the strangeness arising from the scalar exchange term is seen to be very small. On the other hand, the total range term is observed to decrease the energies of the $\mathrm{D}$ and $\bar{D}$ mesons appreciably with increase in the strangeness fraction of the medium, dominantly due to the attractive $d_{1}$ term. Hence, with increase in the strangeness fraction of the medium, the Weinberg Tomozawa term as well as the total range term decrease the energy of $\bar{D}$ mesons, whereas for the D mesons, the Weinberg Tomozawa term leads to an increase in the energy with strangeness and hence partly compensates the decrease due to increase in $f_{s}$ arising from the total range term. This leads to a larger drop of the mass of the $\bar{D}$ mesons as compared to the $\mathrm{D}$ mesons with increase in the strangeness in the medium.

In the present calculations, we observe a drop in the masses of the $D$ as well as $\bar{D}$ mesons. The mass drop is seen to be larger in the presence of hyperons in the medium, as can be observed from Figs. 11 and 2, A drop in the masses of the $D$ mesons was also observed by the recent calculations in the coupled channel approach based on heavy quark symmetry [48]. However, the drop in the $D$ meson mass in Ref. [48] is seen to be much smaller than the findings of the present investigation. The possibility of the formation of D-mesic nuclei arising from an attractive interaction of D-mesons in the nuclear medium has also been investigated in Ref. [48]. In the present investigation, for symmetric nuclear matter at zero temperature, we obtain the mass shifts of about -77 and $-27 \mathrm{MeV}$ for the $D^{+}$and $D^{-}$mesons. The mass shift of the $D$ meson may be compared with the value of $-60 \mathrm{MeV}$ from calculations using quark meson coupling (QMC) model and of $-50 \mathrm{MeV}$ using the QCD sum rule approach [28]. In Ref. [45], the in-medium properties of open charm $D^{+}$and $D^{-}$mesons have been studied in a self-consistent manner using coupled channel approach in cold symmetric nuclear medium. The mass shifts of both the $D^{+}$and $D^{-}$were observed to be repulsive [45], with values of 30 and $18 \mathrm{MeV}$ at nuclear matter saturation density.

In the above, we have investigated the energies of the $\mathrm{D}$ and $\bar{D}$ mesons at zero momentum in the hot asymmetric strange hadronic medium. We then study the effect of the finite momentum on the in-medium energies of the $D$ and $\bar{D}$ mesons. In Fig. 3] we show the optical potentials of $D\left(D^{+}\right.$and $\left.D^{0}\right)$ mesons as functions of momentum, $|\vec{k}|$, at baryon densities, $\rho_{0}$ and $4 \rho_{0}$ for $\eta=0.5$ and $f_{s}=0.5$ and compared to the situation for zero strangeness in the medium. The medium modification of the masses of the $\mathrm{D}$ mesons are reflected in their optical potentials. The larger drop of the mass of the $D^{+}$meson as compared to the $D^{0}$ meson is reflected in their optical potentials and the strangeness is observed to give a larger value for the magnitude of the optical potentials which reflects the fact that the mass drop of $\mathrm{D}$ mesons is larger with increase in strangeness in the medium as has already been illustrated in Fig. 1 .

Fig. 4 shows the optical potentials of $\bar{D}$ mesons as functions of momentum, $|\vec{k}|$ at baryon densities $\rho_{0}$ and $4 \rho_{0}$, which reflect the medium modifications of the masses of the $\bar{D}$ mesons as shown in Fig. 1. The optical potentials of $\bar{D}$ mesons also reflect the larger sensitivity of the mass of the $\bar{D}^{0}$ meson to the strangeness content of the medium as compared to the mass of the $D^{-}$meson. The dependence of the optical potentials of $\bar{D}^{0}$ meson on strangeness of the medium is seen to be less at finite temperatures as compared to the case of zero temperature. This is related to the fact that the masses of the $D$ and $\bar{D}$ mesons have smaller drop in the medium as we increase the temperature. The momentum dependence of the optical potentials of the $\mathrm{D}$ and $\bar{D}$ mesons are observed to be small for small values of $\mathrm{k}$. This is due to the reason that when $k^{2}$ is small as compared to $m_{D}{ }^{2}$ or $m_{D}{ }^{* 2}$, the optical potential given by equation (22) can be approximated as $\left(m_{D}{ }^{*}-m_{D}\right)\left(1-k^{2} /\left(2 m_{D}{ }^{*} m_{D}\right)\right)$, where the second term with the momentum 

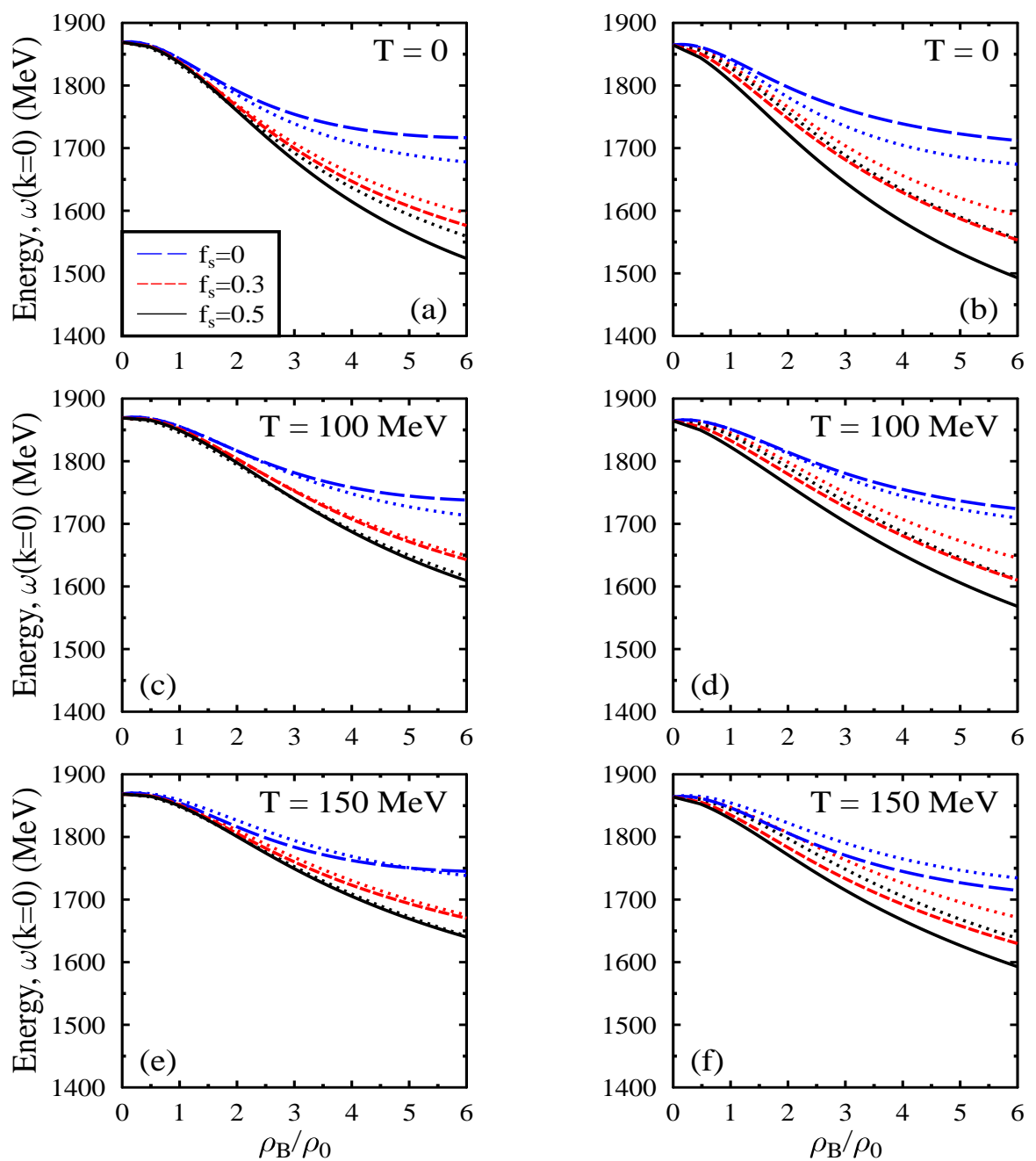

FIG. 2: (Color online)The energies of $D^{-}$(subplots a, c, e) and $\bar{D}^{0}$ (subplot b, d, f) mesons at zero momentum in isospin asymmetric $(\eta=0.5)$ hyperonic medium at different temperatures, plotted as functions of baryon density in units of nuclear matter saturation density, $\rho_{B} / \rho_{0}$, shown for various values of the strangeness fraction, $f_{s}$. The results are compared with the case of isospin symmetric hadronic matter $(\eta=0)$ shown as dotted lines.

dependence is small compared to 1 and hence for small $\mathrm{k}$, the momentum dependence is observed to be small for the optical potential. However, one observes the momentum dependence to be larger for higher values of $\mathrm{k}$, as can be seen from Figs. 3 and 4 .

Due to the different masses of the $D^{+}$and $D^{0}$ mesons, as well as of the $D^{-}$and $\overline{D^{0}}$ mesons, in the isospin asymmetric hadronic medium, and due to the modifications of the masses of the charmonium states, the production cross sections, yield and the collective flow are expected to be different for the $D^{+}$and $D^{0}$, as well as, for the $D^{-}$and $\bar{D}^{0}$ mesons in the isospin asymmetric hadronic environment. The present investigation shows a stronger isospin dependence of the masses of the $D^{+}$and $D^{0}$, as compared to the masses of the $D^{-}$and $\bar{D}^{0}$ mesons, particularly at high densities and this is observed to be more dominant at higher values of the strangeness fraction. This should show up in the 

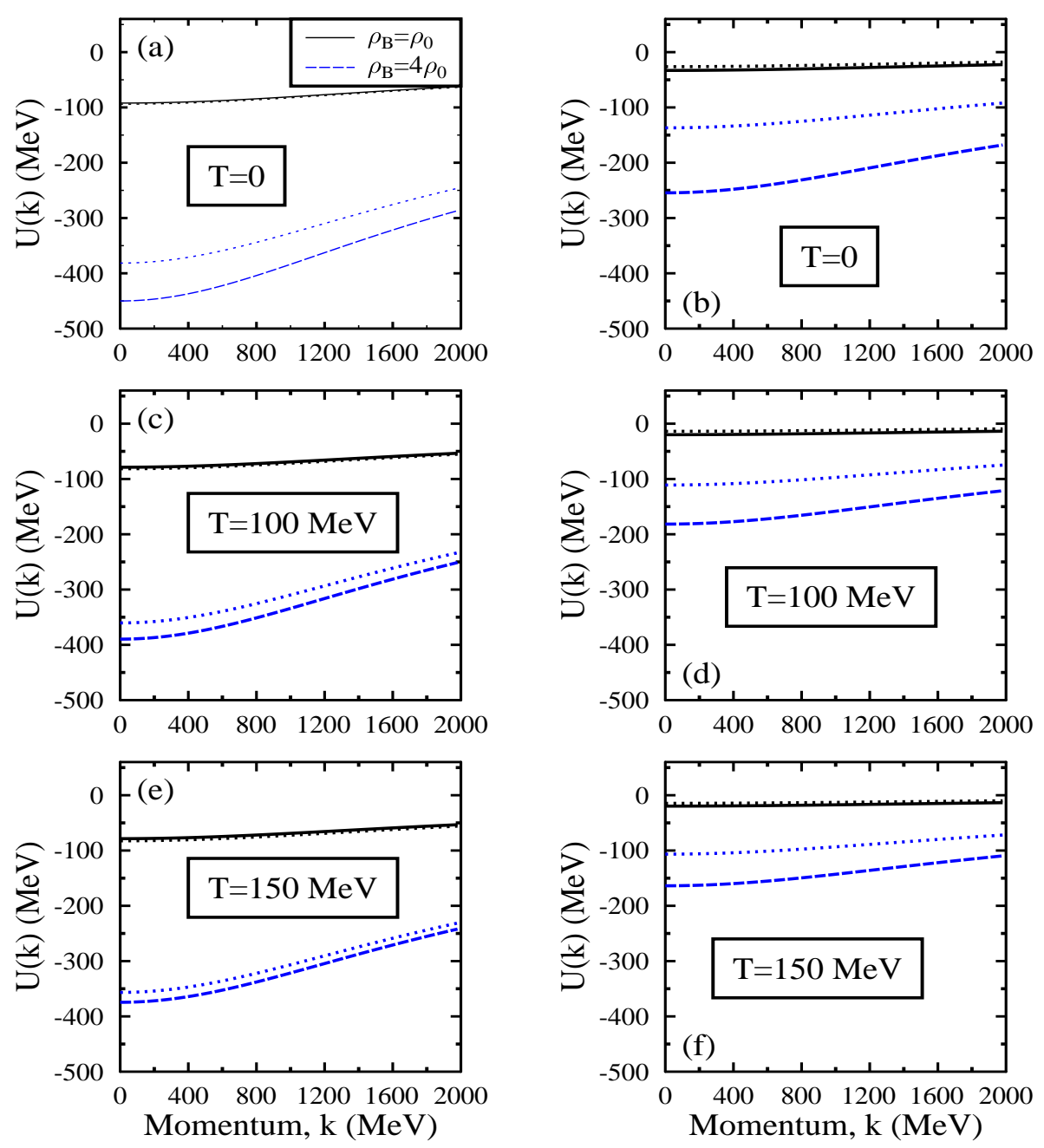

FIG. 3: (Color online) The optical potentials of the $D$ mesons (subplots (a), (c) and (e) are for $D^{+}$mesons and (b), (d), and (f) are for $D^{0}$ mesons), plotted as functions of momentum, $\mathrm{k}(\mathrm{MeV})$, at baryon densities of $\rho_{B}=\rho_{0}$ and $4 \rho_{0}$, for $\eta=0.5$ and $f_{s}=0.5$. The results are compared with the case of $f_{s}=0$ shown as dotted lines.

experimental observables of the ratios of $D^{+} / D^{0}$ as well as $D^{-} / \overline{D^{0}}$ in the production cross-section as well as in the parameter, $v_{2}$ for the collective flow for the ratio $D^{+} / D^{0}$ from the high density matter resulting from compressed baryon matter (CBM) experiment at the FAIR project in the future facility at GSI. However, the $D$ and $\bar{D}$ mesons, due to the presence of the light antiquark (quark) can undergo substantial decay within the hadronic environment, and this could make it difficult to get clean signals from the experimental data from the heavy ion collision experiments. 

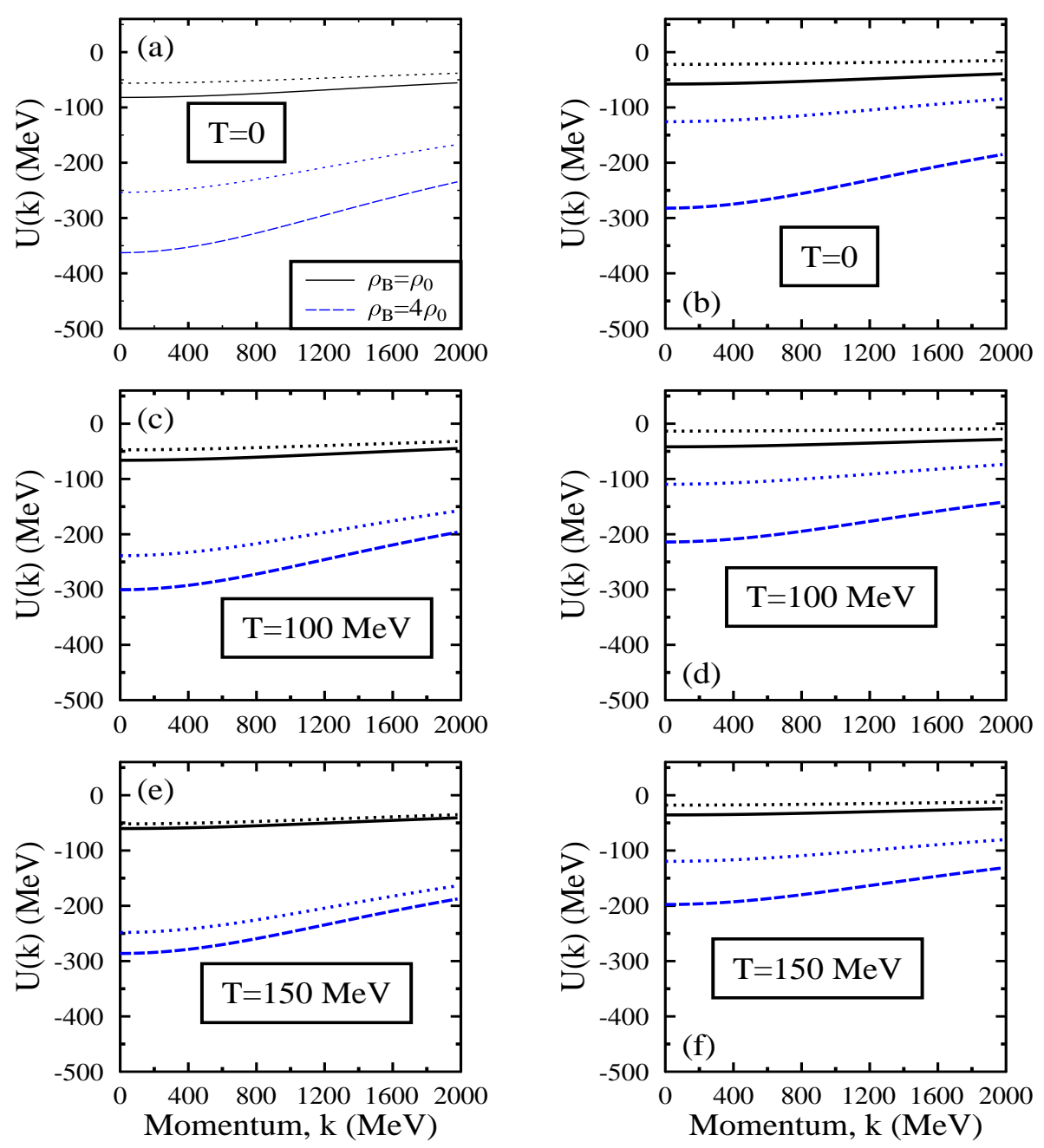

FIG. 4: (Color online)The optical potentials of the $\bar{D}$ mesons (subplots (a), (c) and (e) are for $D^{-}$mesons and (b), (d), and (f) are for $\bar{D}^{0}$ mesons) plotted as functions of momentum, $\mathrm{k}(\mathrm{MeV})$, at baryon densities of $\rho_{B}=\rho_{0}$ and $4 \rho_{0}$, for $\eta=0.5$ and $f_{s}=0.5$. The results are compared with the case of $f_{s}=0$ shown as dotted lines.

\section{B. Charmonium states in the hadronic matter}

We then investigate how the behavior of the dilaton field $\chi$ in the hot asymmetric strange hadronic matter affects the in-medium masses of the charmonium states $J / \psi, \psi(3686)$ and $\psi(3770)$. In Fig. 5 we show the shifts of the masses of the charmonium states $J / \psi, \psi(3686)$ and $\psi(3770)$ from their vacuum values, in the isospin asymmetric hyperonic matter $\left(\eta=0.5, f_{s}=0.5\right)$. These are plotted as functions of the baryon density for values of temperature, $\mathrm{T}$ $=0,100$ and $150 \mathrm{MeV}$. For these temperatures, in the subplots (a), (c) and (e) the mass shifts in the charmonium states are shown when the gluon condensate in the medium is calculated with the finite quark mass term taken into account in the trace of the energy momentum tensor and the subplots (b), (d) and (f) show the results while neglecting the quark mass term. The values of the mass shift of $J / \psi$ for the isospin symmetric medium at zero temperature are 

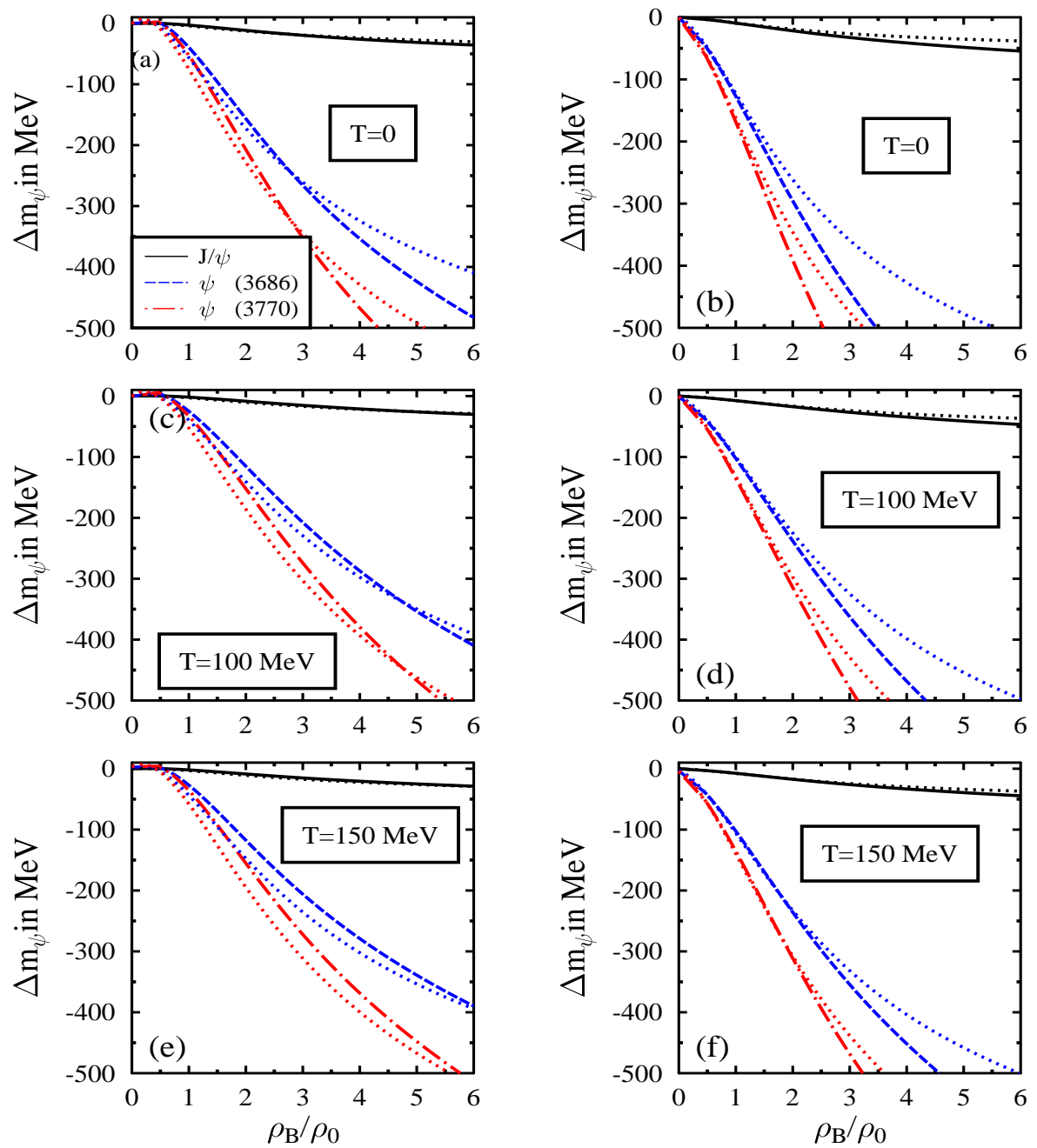

FIG. 5: (Color online) The mass shifts of the charmonium states, $J / \psi, \psi(3686)$ and $\psi(3770)$ mesons in isospin asymmetric hyperonic matter $\left(\eta=0.5\right.$ and $\left.f_{s}=0.5\right)$, plotted as functions of baryon density in units of nuclear matter saturation density, $\rho_{B} / \rho_{0}$, for values of temperature, $\mathrm{T}=0,100$ and $150 \mathrm{MeV}$. The mass shifts are shown in subplots (a), (c) and (e), when the gluon condensate in the medium is calculated by accounting for the finite quark mass term in the trace of the energy momentum tensor, and in subplots (b), (d) and (f), when the quark mass term is neglected. The results are compared with the case of asymmetric nuclear matter $\left(f_{s}=0\right)$, shown as dotted lines.

found to be $-4.35(-2.96) \mathrm{MeV}$ and $-26.37(-25.95) \mathrm{MeV}$ for $f_{s}=0(0.5)$ at $\rho_{B}=\rho_{0}$ and $4 \rho_{0}$ respectively, and these values are modified to $-4.16(-2.9) \mathrm{MeV}$ and $-23.9(-26.06) \mathrm{MeV}$ for $\eta=0.5$. These mass shifts are for the situation when the finite quark mass term is taken into account in the trace anomaly and hence the gluon condensate which is calculated is a combined effect of the modifications of the scalar dilaton field as well as the quark condensates through the scalar $\sigma$ and $\zeta$ fields. One notices that the effects of temperature are small on the masses of the charmonium states. In isospin symmetric hadronic medium $(\eta=0)$ at zero temperature, at baryon density, $\rho_{B}=\rho_{0}$ and the value of the strangeness fraction $f_{s}=0(0.5)$, the mass shifts for the charmonium states, $\psi(3686)$ and $\psi(3770)$ are observed to be $-59(-40) \mathrm{MeV}$ and $-78.5(-54) \mathrm{MeV}$ respectively, and these values are modified to $-365.4(-352) \mathrm{MeV}$ and 

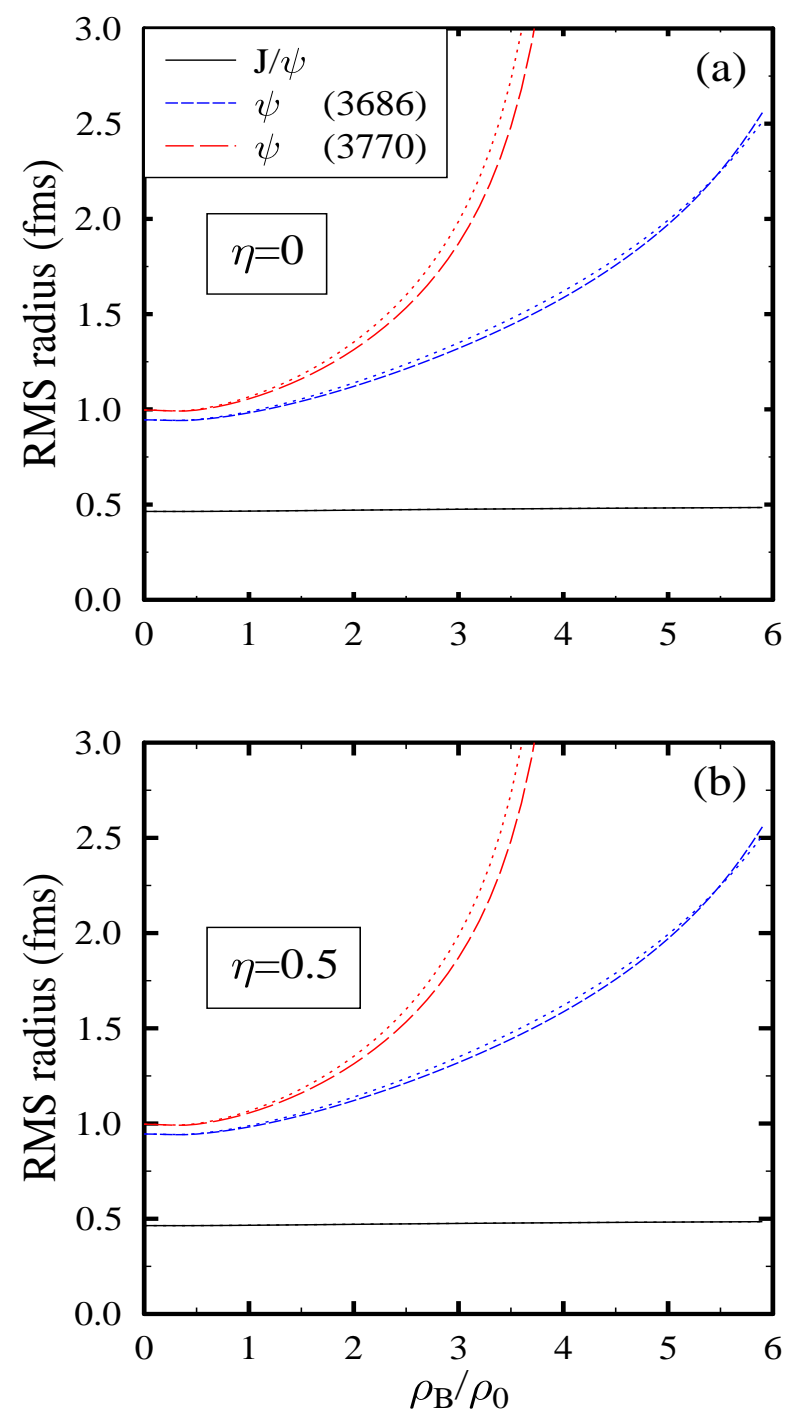

FIG. 6: (Color online) The rms radii of the charmonium states, $J / \psi, \psi(3686)$ and $\psi(3770)$ plotted as functions of baryon density in units of nuclear matter saturation density, $\rho_{B} / \rho_{0}$, for $f_{s}=0.5$ and zero temperature. These have been shown in subplots (a) and (b) for the isospin symmetric $(\eta=0)$ and isospin asymmetric hyperonic matter (with $\eta=0.5)$. The results are compared with the case of asymmetric nuclear matter $\left(f_{s}=0\right)$, shown as dotted lines.

$-483.4(-466) \mathrm{MeV}$ for $\rho_{B}=4 \rho_{0}$. One observes that the effects of the strangeness, isospin asymmetry as well as temperature on the mass modifications of the charmonium states in the hadronic medium are small and the dominant medium effect is observed to be the effect of density, in the present investigation.

As already mentioned, in obtaining the mass shifts of the charmonium states as discussed above, we have taken into account the finite quark mass term in the trace of the energy momentum tensor and hence in the evaluation of the gluon condensate in the medium. However, if we do not consider the finite quark mass term in the trace of the energy momentum tensor and calculate the mass shift of the charmonium states, then in the isospin symmetric medium, at nuclear saturation density, $\rho_{B}=\rho_{0}$ and strangeness fraction $f_{s}=0(0.5)$, the values of the mass shifts for the charmonium states $J / \psi, \psi(3686)$ and $\psi(3770)$ are observed to be $-9.32(-9.07),-126.4(-123)$ and $-167.5(-163) \mathrm{MeV}$ 
respectively, for $N_{f}=4$ in the expression of the one loop QCD beta function given by equation (4) and $-8.63(-8.41)$, $-117(-114)$ and $-155(-151)$, when we take $N_{f}=3$ in the beta function, to obtain the expression for the gluon condensate. For the limit of massless quarks, the difference in the mass shifts of the charmonium masses arises due to the different multiplying factors in the equation relating the scalar gluon condensate and the dilaton field. There is seen to be a larger mass shift for $N_{f}=4$, due to the factor $(24 / 25)$ in equation (5), which is larger than the factor (8/9) for the case of $N_{f}=3$. The values of the mass shifts of these charmonium states in isospin symmetric matter at zero temperature for $\rho_{B}=\rho_{0}$ in the limit of massless quarks obtained for $N_{f}=4$, may be compared to the values of $-4.35(-2.96),-59(-40)$ and $-78.5(-54) \mathrm{MeV}$, when the finite quark mass term in the trace of the energy momentum tensor is taken into account. The effects of the finite quark mass on the masses of $J / \psi$ and $\eta_{c}$ obtained using the QCD sum rule approach due to the medium modifications of the gluon condensates within the present chiral effective model, has been considered in Ref. [75]. In Ref. [75], the value of the mass of $J / \psi$, in symmetric nuclear matter at zero temperature for $\rho_{B}=\rho_{0}$, was obtained to be $-4.48(-8.01) \mathrm{MeV}$, with (without) accounting for the finite quark masses in the trace of the energy momentum tensor, which may be compared with the value of mass shift of $J / \psi$ as $-4.35(-9.32) \mathrm{MeV}$ in the present investigation. In the limit of massless quarks, the values of mass shifts of the charmonium states $J / \psi, \psi(3686)$ and $\psi(3770)$, are observed to be $-34.72(-39.82),-471(-540.5)$ and $-623(-714)$ $\mathrm{MeV}$ respectively, in symmetric hadronic matter for $f_{s}=0(0.5)$, for baryon density $4 \rho_{0}$. In isospin asymmetric medium $(\eta=0.5)$, at $\rho_{B}=\rho_{0}$ and $f_{s}=0(0.5)$, these values are observed to be $-9.02(-9.37),-122.5(-127)$ and $-162(-168) \mathrm{MeV}$. Thus we observe that the magnitudes of the mass shifts for the charmonium states are smaller when we consider the contribution of finite quark mass term for the modification of the gluon condensate in the medium. The medium modification of the gluon condensate is given in terms of the medium modifications of the dilaton field and the scalar fields, $\sigma, \zeta$ and $\zeta_{c}$, as can be seen from equation (27). As we have already mentioned, the fluctuation of the charm scalar field $\zeta_{c}$ has been neglected in the present investigation for the study of the medium modifications of the $D, \bar{D}$ mesons as well as for the study of the in-medium masses of the charmonium states. The contribution of terms containing the scalar fields $\sigma$ and $\zeta$ arising from the finite quark mass term in the trace of the energy momentum tensor to the medium modification of the gluon condensate given by equation (27), are opposite in sign as compared to the first term given in terms of the scalar dilaton field and this leads to the charmonium mass shifts to be smaller when the finite quark mass term is accounted for in the trace anomaly as compared to when it is neglected.

In Ref. [55], the mass modifications of the charmonium states were calculated in the symmetric nuclear medium at zero temperatures using QCD second order Stark effect in the linear density approximation. The mass shifts for the charmonium states $J / \psi, \psi(3686)$ and $\psi(3770)$ were found to be $-8,-100$ and $-140 \mathrm{MeV}$ respectively at the nuclear matter saturation density. These may be compared to the values of $-4.35,-59$ and $-78.5 \mathrm{MeV}$ of the charmonium states at $\rho_{B}=\rho_{0}$ of our present calculations when the effects of the finite quark masses are taken into account in evaluation of the medium modification of the gluon condensate and the values of $-9.32,-126.4$ and $-167.5 \mathrm{MeV}$, in the limit of massless quarks in the trace of energy momentum tensor in QCD. In the present work, we have studied the isospin asymmetry and strangeness dependence of the masses of the charmonium states in the hadronic medium at finite temperatures, which will be relevant for the asymmetric heavy ion collision experiments planned at the future facility at GSI. Using QCD sum rules and operator product expansion up to mass dimension four [83] and six [90], the mass shift for $J / \psi$ mesons at nuclear saturation density was observed to be about $-7 \mathrm{MeV}$ and $-4 \mathrm{MeV}$ respectively. The effect of temperature on the $J / \psi$ in deconfinement phase was studied in [89, 91] and it was reported that $J / \psi$ mass remains essentially constant within a wide range of temperature and above a particular value of the temperature, $\mathrm{T}$, there is seen to be a sharp change in the mass of $J / \psi$ in the deconfined phase. For example, in Ref. [92] the mass shift for $J / \psi$ was reported to be about $200 \mathrm{MeV}$ at $\mathrm{T}=1.05 \mathrm{~T}_{c}$. In the present work, we have studied the effects of temperature, density, isospin asymmetry and strangeness fraction, on the mass modifications of the charmonium states $(J / \psi, \psi(3686)$ and $\psi(3770))$ in the confined hadronic phase, arising due to modifications of a scalar dilaton field which simulates the gluon condensates of QCD, as well as due to fluctuations of the scalar fields $\sigma$ and $\zeta$ in the explicit chiral symmetry breaking term, within the chiral effective model, when the finite quark masses are taken into account in the trace of the energy momentum tensor of QCD. The effect of temperature is found to be small for the charmonium states $J / \psi, \psi(3686)$ and $\psi(3770)$, whereas the masses of charmonium states are observed to vary considerably with density, in the present investigation. In Ref. [55] the masses of the charmonium states have been studied due to the effects of the $D \bar{D}$ loop as well as of from the gluon condensates in the medium and the mass shifts for $J / \psi, \psi(3686)$ and $\psi(3770)$ mesons were observed to be $-5,-130$ and $-125 \mathrm{MeV}$ at nuclear saturation density in symmetric cold nuclear medium, with the medium modification of the charmonium masses arising from the modification of the gluon condensate dominating over the contribution from the $\mathrm{D}$ meson loop. On the other hand, considering the effects of $D \bar{D}^{*}, D^{*} \bar{D}$ and $D^{*} \bar{D}^{*}$ meson loops, in addition to the $D \bar{D}$ loop, with the medium modifications of the $\mathrm{D}$ and $D^{*}$ mesons calculated in a Quark meson coupling model, the mass shift for $J / \psi$ mesons at 
nuclear matter density was observed to range from $-16 \mathrm{MeV}$ to $-24 \mathrm{MeV}$ [58], with the contribution from the $D^{*} \bar{D}^{*}$ loop dominating over the other terms. The range of the mass shifts of $J / \psi$ was obtained depending on the value of the ultra-violet cut-off in the form factors used to regularize the loop integrals.

The strength of the harmonic oscillator wave function, $\beta$ can be modified in the hadronic matter, which can give an estimate of the change in the size of the charmonium state in the medium. The shift in $\beta^{2}$ can be obtained by relating to the mass shift of the charmonium state given as $\delta M_{J / \psi}=\frac{3}{2 M} \delta \beta^{2}$ for $J / \psi[57]$ and $\delta M_{\psi}=\frac{7}{2 M} \delta \beta^{2}$ for the charmonium states $\psi(3686)$ and $\psi(3770)$. We compute the root mean squared radii of the charmonium states from the mass shifts obtained in the present work, at given densities, and show the obtained results in Fig. 6 for the isospin symmetric $(\eta=0)$ and asymmetric (with $\eta=0.5)$ strange hadronic matter. These results are compared to the case of nuclear matter $\left(f_{s}=0\right)$, shown as dotted lines. We observe that the change in the radius of the charmonium state $J / \psi$ from the vacuum value of $0.47 \mathrm{fms}$ is marginal, even for high densities. For example,the radius of $J / \psi$ is modified to about $0.484 \mathrm{fms}$ for $\rho_{B}=6 \rho_{0}$. On the other hand, it is observed that the sizes of the charmonium states $\psi(3686)$ and $\psi(3770)$, are seen to increase substantially at higher densities as can be seen in Fig. 6. This is related to the fact that the mass shifts for these mesons are large at high densities in the medium. The value of the rms radii for $\psi(3770)$ is seen to rise faster than that of the $\psi(3686)$ at high densities.

The medium modifications of the masses of $D$ and $\bar{D}$ mesons can have relevance to the experimental observable of $J / \psi$ suppression in relativistic heavy ion collision experiments. Due to the drop in the mass of the $D \bar{D}$ pair in the hadronic medium, it can become a possibility that the excited states of charmonium $\psi(3686)$ and $\psi(3770)$ can decay to $D \bar{D}$ pairs [74] and hence the production of $J / \psi$ from the decay of these excited states can be suppressed. Even at some densities it can become a possibility that the $J / \psi$ itself decays to $D \bar{D}$ pairs. The effects of the medium modifications of the $D(\bar{D})$ mesons on the decay widths for the decay of the charmonium states to $D \bar{D}$ pairs has been studied accounting for the internal structure of these mesons using the 3P0 model [57]. In this model, it was observed that the decay width does not increase monotonically with drop in the masses of the $D(\bar{D})$ mesons with density as one would naively expect. On the contrary, the decay widths after an increase initially with decrease in the $\mathrm{D}(\bar{D})$ meson masses was seen to decrease with further drop in these masses and nodes were observed in the decay widths of the charmonium states $\psi(3686)$ and $\psi(3770)$ [57]. In the present investigation, we study the effects of the mass modifications of the $D(\bar{D})$ mesons as well as of the charmonium states in the isospin asymmetric hyperonic medium on the partial decay widths of the charmonium states to $D^{+} D^{-}$as well as $D^{0} D^{0}$ pairs. We also observe nodes in the partial decay widths of the charmonium states $\psi(3686)$ and $\psi(3770)$, as has already been observed in the literature [57], when the mass modifications of the $D(\bar{D})$ mesons are taken into account, but the mass modifications of the charmonium masses are neglected. However, there are observed to be significant modifications to these partial decay widths, when the mass modifications of the charmonium states are also taken into account. There are no nodes observed even up to a density of about $6 \rho_{0}$, when the in-medium masses of the charmonium states are taken into account in addition to the in-medium $D$ and $\bar{D}$ meson masses.

We next compare the in-medium masses of the charmonium states and the $D \bar{D}$ pair to investigate the possibility of the decay of the charmonium states to $D \bar{D}$ in the hadronic medium. In Fig. 7 , we show the density dependence of the masses of the $D^{+} D^{-}$as well as $D^{0} \bar{D}^{0}$ pairs at strangeness fraction, $f_{s}=0.5$ and for isospin asymmetry parameter, $\eta=0.5$. These are shown for values of temperatures, $\mathrm{T}=0,100$ and $150 \mathrm{MeV}$. In the same figure, we also show the in-medium masses of the charmonium states $J / \psi, \psi(3686)$ and $\psi(3770)$ considering the contribution of finite quark mass term in the trace of the energy momentum tensor in QCD. We compare these results with the case of $f_{s}=0$ shown as dotted lines. The masses of $D$ and $\bar{D}$ mesons are observed to decrease with inclusion of hyperons in the medium, as has already been observed in Figs. 1 and 2, and hence with increase in $f_{s}$, there is a drop in the mass of the $D \bar{D}$ pair. However, there is seen to be a larger drop of the mass of $D^{0} \bar{D}^{0}$ as compared to $D^{+} D^{-}$pair in the isospin asymmetric medium. The masses of the charmonium states are also observed to decrease with increase in $f_{s}$. The possibility of the decay of charmonium states to $D^{+} D^{-}$as different from the decay to $D^{0} \bar{D}^{0}$ pair in the isospin asymmetric medium, is thus due to the difference in the mass modifications of the $D^{+} D^{-}$and $D^{0} \bar{D}^{0}$ pairs in the hadronic medium. We observe from Fig. 7 that the in-medium mass of the excited charmonium state, $\psi(3770)$ always remains larger than the in-medium masses of $D^{+} D^{-}$pairs in the asymmetric hadronic matter $(\eta=0.5)$ for both $f_{s}=0$ and 0.5 and hence it can decay to $D^{+} D^{-}$pairs at all values of densities. However, it is observed that the mass of $\psi(3770)$ remains larger than $D^{0} \overline{D^{0}}$ pair for densities smaller than a density of around $2.8 \rho_{0}$ for $\eta=0.5$ and $f_{s}=0$, and hence the decay of $\psi(3770)$ to $D^{0} \bar{D}^{0}$ is possible only for densities less than this density. However, for the asymmetric strange hadronic matter $\left(\eta=0.5, f_{s}=0.5\right)$, the decay of $\psi(3770)$ to $D^{0} \bar{D}^{0}$ is a possibility for all densities. The mass of $\psi(3686)$ is observed to be larger than the in-medium mass of $D^{+} D^{-}$pairs above a baryon density of about $0.8 \rho_{0}\left(0.5 \rho_{0}\right)$ for $f_{s}=0(0.5)$ and hence it is a possibility that this state can decay to $D^{+} D^{-}$pairs above this value of density in the asymmetric hadronic matter. The decay of these excited charmonium states to $D \bar{D}$ pairs may lead to $J / \psi$ suppression. The $J / \psi$ mass is seen to be larger than the mass of the $D^{+} D^{-}\left(D^{0} \bar{D}^{0}\right)$ pair above 

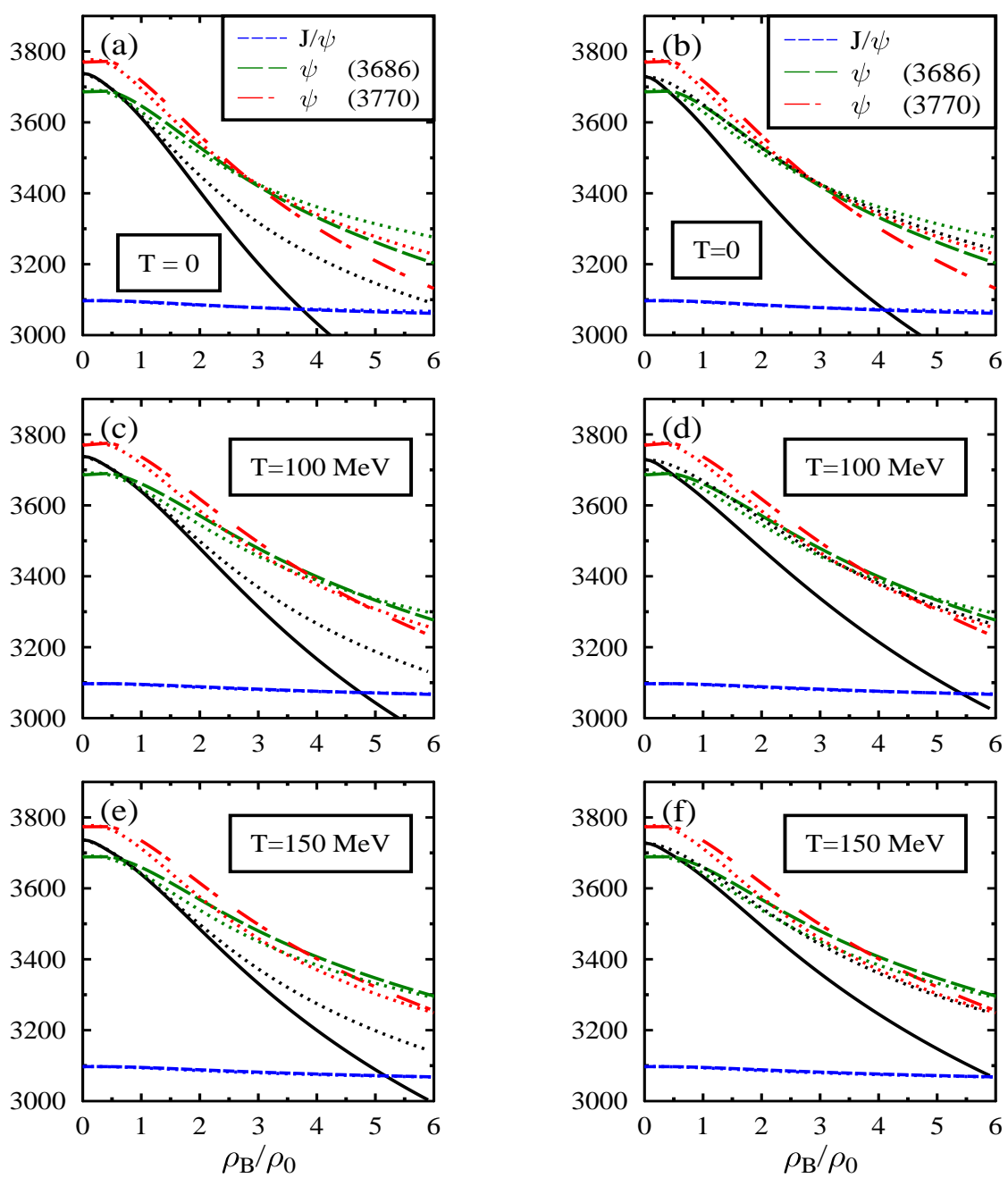

FIG. 7: (Color online) The masses of the charmonium states as well as the $D \bar{D}$ pair (shown by the solid line) $\left[D^{+} D^{-}\right.$in (a), (c), (e) and $D^{0} \bar{D}^{0}$ in (b), (d), (f)] in $\mathrm{MeV}$ plotted as functions of $\rho_{B} / \rho_{0}$ for isospin asymmetric strange hadronic matter $\left(\eta=0.5, f_{s}=0.5\right)$ and for temperatures, $\mathrm{T}=0,100,150 \mathrm{MeV}$. The charmonium masses have been calculated from the gluon condensate in the medium, obtained by accounting for the finite masses of the quarks. These results have been compared with the case of $f_{s}=0$ shown as dotted lines.

a density of $3.8 \rho_{0}\left(4 \rho_{0}\right)$ in the isospin asymmetric strange hadronic matter $\left(\eta=0.5, f_{s}=0.5\right)$ at zero temperature, which can lead to the decay of $J / \psi$ to $D \bar{D}$ pairs at high densities. The density above which there can be possibility of the decay of $J / \psi$ to $D \bar{D}$ pairs is seen to increase as one increases the temperature of the hadronic medium. In Fig. 7 we have investigated the medium modifications of the charmonium masses in the strange hadronic matter, accounting for the finite quark mass term in the trace of the energy momentum tensor while evaluating the scalar gluon condensate in the medium. On the other hand, if we do not consider the contribution of finite quark masses to the in-medium properties of charmonium states, then there is seen to be a much larger drop of the charmonium masses, which considerably modifies the conclusions for the possibility of the decay of the charmonium states to $D \bar{D}$ pairs. We show these results in Fig. 8 . For $\eta=0.5$ and $f_{s}=0.5$, the charmonium state $\psi(3770)$ is observed to have a 

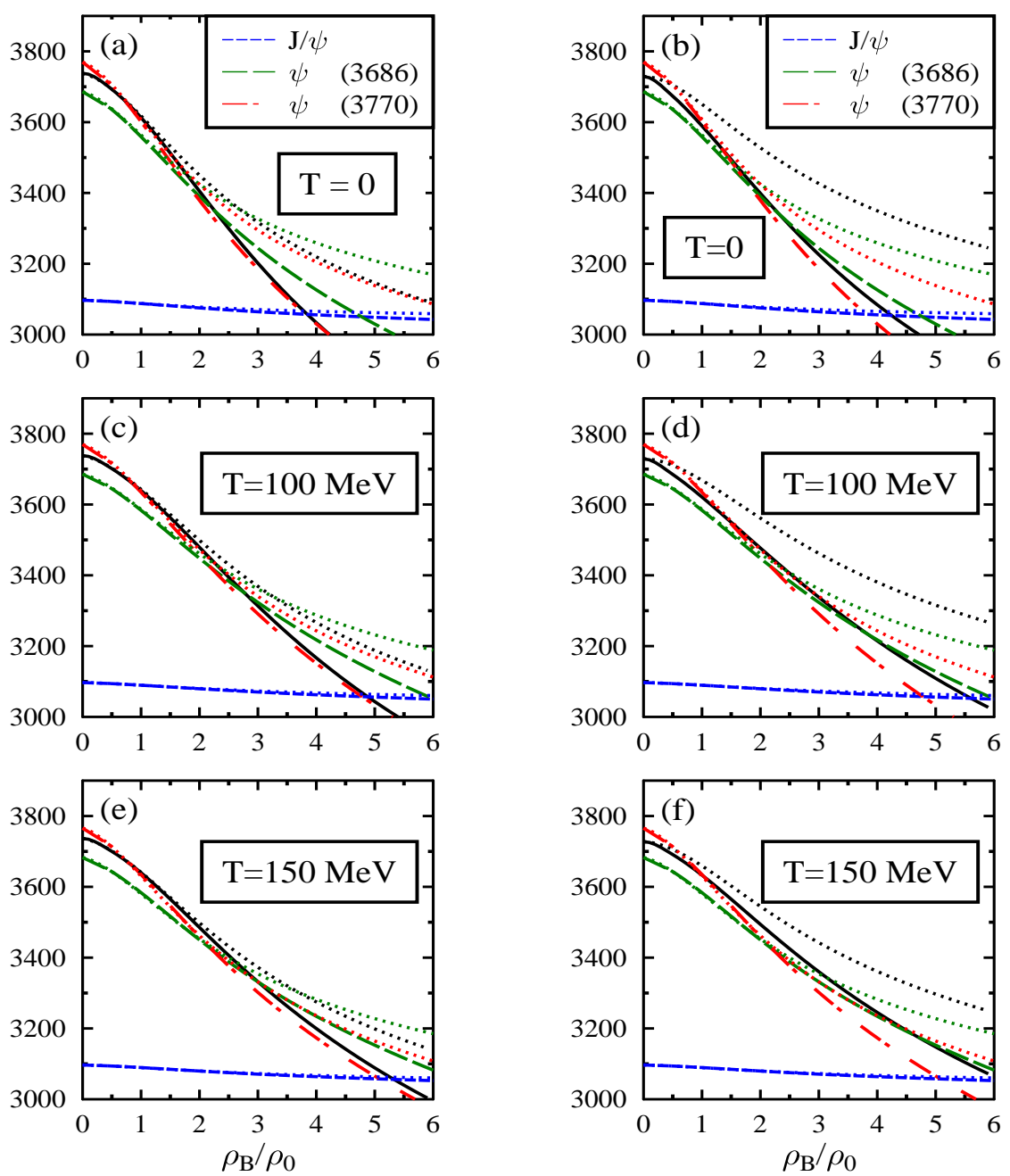

FIG. 8: (Color online) The masses of the charmonium states as well as the $D \bar{D}$ pair (shown by the solid line) $\left[D^{+} D^{-}\right.$in (a), (c), (e) and $D^{0} \bar{D}^{0}$ in (b), (d), (f)] in $\mathrm{MeV}$ plotted as functions of $\rho_{B} / \rho_{0}$ for isospin asymmetric strange hadronic matter $\left(\eta=0.5, f_{s}=0.5\right)$ and for temperatures, $\mathrm{T}=0,100,150 \mathrm{MeV}$. The charmonium masses have been calculated from the gluon condensate in the medium, obtained by neglecting the finite masses of the quarks in the trace anomaly. These results have been compared with the case of $f_{s}=0$, shown as dotted lines.

mass larger than the mass of the $D^{+} D^{-}\left(D^{0} \overline{D^{0}}\right)$ pair for a density smaller than $0.8 \rho_{0}\left(1.4 \rho_{0}\right)$, and hence the decay to $D^{+} D^{-}\left(D^{0} \bar{D}^{0}\right)$ can be a possibility for densities smaller than this density. The charmonium state $\psi(3686)$ can decay to $D^{+} D^{-}\left(D^{0} \overline{D^{0}}\right)$ for densities above a density of around $2.2 \rho_{0}$. For $J / \psi$, the decay to $D^{+} D^{-}\left(D^{0} \bar{D}^{0}\right)$ seems possible above a density of around $3.8 \rho_{0}\left(4.2 \rho_{0}\right)$, when one does not account for the quark mass term in the trace anomaly, for the computation of the charmonium masses from the medium modification of the gluon condensate.

The possibility of the decay of the charmonium states to the $D \bar{D}$ pairs has been investigated in isospin asymmetric nuclear matter at finite temperature within the model used in the present investigation [70]. However, in this work [70], the effect of the finite quark masses were neglected in the trace of the energy momentum tensor for the evaluation of the gluon condensate modifying the masses of the charmonium states in the medium. As we have already discussed, 

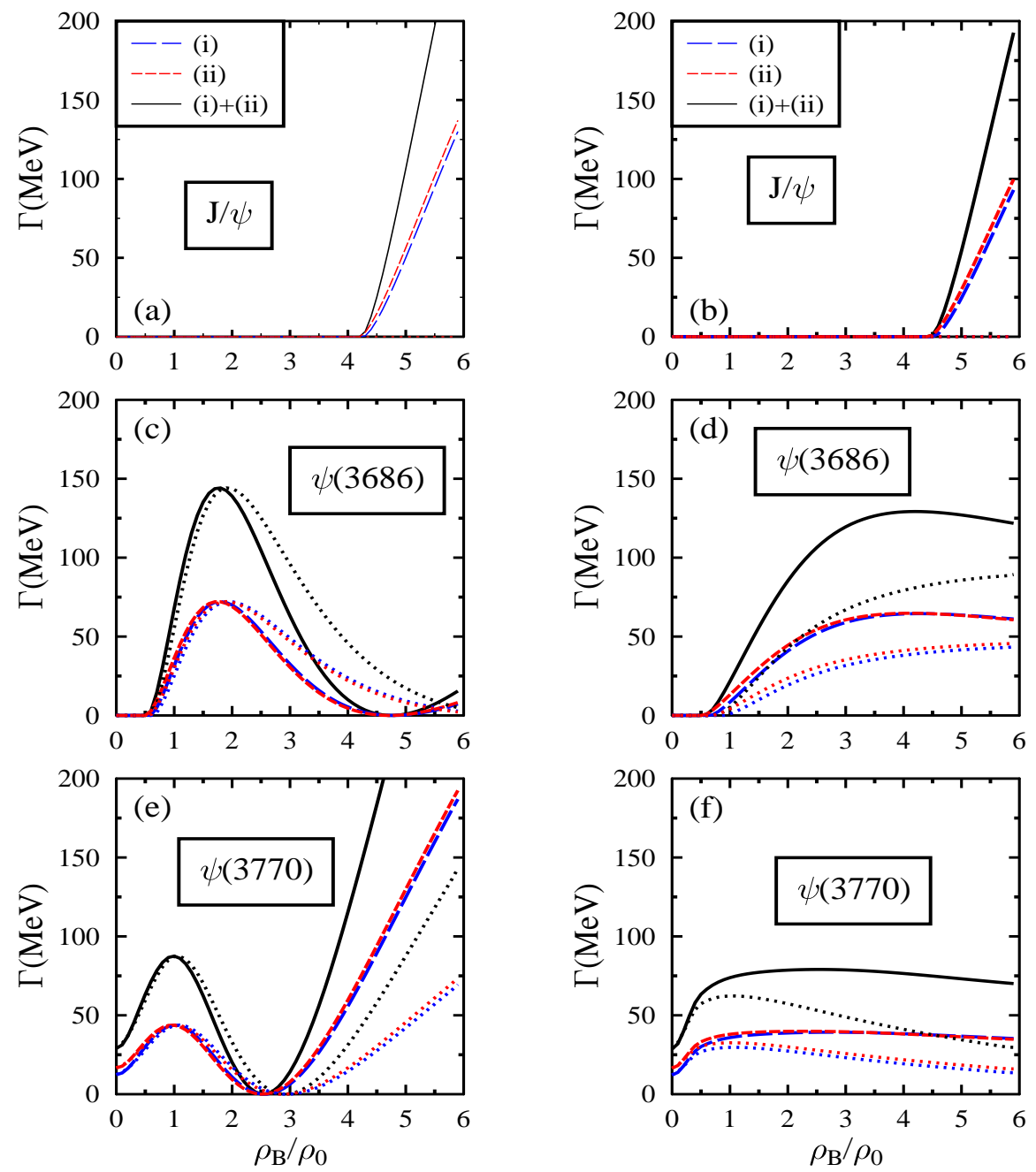

FIG. 9: (Color online) The partial decay widths of the charmonium states to (i) $D^{+} D^{-}$, (ii) $D^{0} \overline{D^{0}}$ and (iii) the sum of the two channels $((\mathrm{i})+(\mathrm{ii}))$ in the isospin symmetric strange hadronic matter $\left(\eta=0, f_{s}=0.5\right)$, accounting for the medium modifications of the $D(\bar{D})$ mesons. These are shown in subplots (a), (c) and (e), when the mass modifications of the charmonium states are neglected and (b), (d) and (f), the partial decay widths are shown when the mass modifications of the charmonium states are also taken into account. These results are compared to the case of $f_{s}=0$, shown as dotted lines.

the charmonium states are observed to have much smaller drop in the medium when the finite quark masses are taken into account leading to a larger possibility of the decay of charmonium states to the $D \bar{D}$ pairs. In Ref. [70], due to the larger drop in the charmonium masses in the limit of massless quarks, as compared to the present investigation, where the finite quark masses are taken into consideration, the charmonium states decaying to the $D \bar{D}$ pairs was becoming a possibility only for high densities, when the charmonium masses were larger than the mass of the $D \bar{D}$ pair. The effect of strangeness on the masses of the $\mathrm{D}$ and $\bar{D}$ mesons in the present investigation is to decrease the masses of the $D$ and $\bar{D}$ mesons as well as of the charmonium states in the massless quark limit. However, while accounting for the finite quark mass term in the trace of energy momentum tensor, the gluon condensate in the medium has contributions from the dilaton field as well as from the scalar $\sigma$ and $\zeta$ fields, which is seen to the observed behavior 

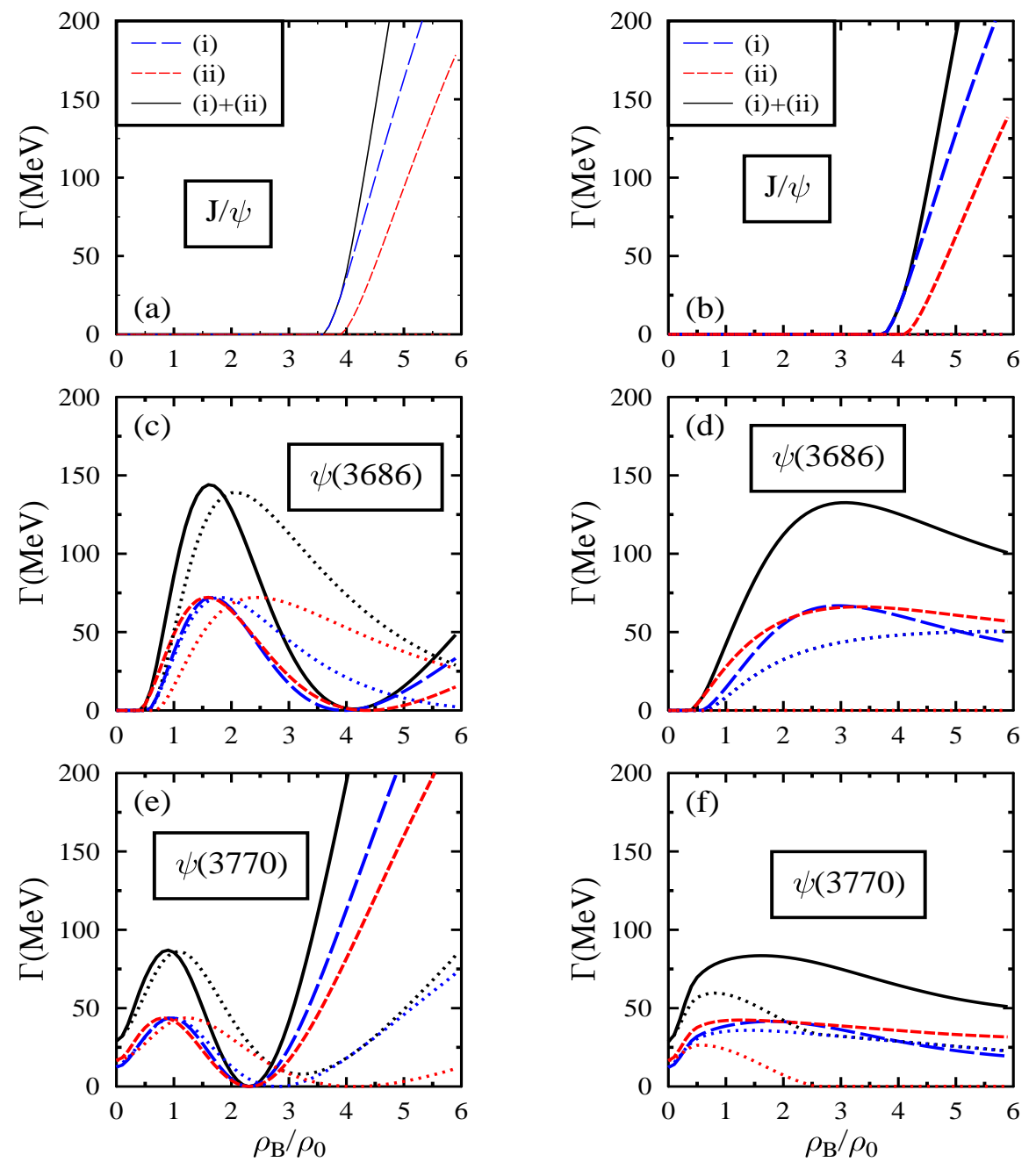

FIG. 10: (Color online) The partial decay widths of the charmonium states to (i) $D^{+} D^{-}$, (ii) $D^{0} \overline{D^{0}}$ and (iii) the sum of the two channels $((\mathrm{i})+(\mathrm{ii}))$ in the isospin asymmetric strange hadronic matter $\left(\eta=0.5, f_{s}=0.5\right)$ at $\mathrm{T}=0$, as functions of the baryon density, in units of the nuclear matter saturation density, accounting for the medium modifications of the $D(\bar{D})$ mesons. These are shown in subplots (a), (c) and (e), when the mass modifications of the charmonium states are neglected and (b), (d) and (f), the partial decay widths are shown when the mass modifications of the charmonium states are also taken into account. These results are compared to the case of $f_{s}=0$, shown as dotted lines.

of smaller drop of the charmonium states in the medium as compared to the case for massless quark limit, as shown in Fig. 5 .

\section{Partial Decay widths of the charmonium states to $D \bar{D}$ pairs}

We compute the partial decay widths of the charmonium states to $D \bar{D}$ pairs by accounting for the internal structure of these mesons using the $3 \mathrm{P} 0$ model. These decay widths are calculated by using the expressions as given by equations 
(30), (31) and (32), which are computed from the matrix element for the specific charmonium state decaying to $D \bar{D}$ pair. The wave functions for the charmonium state as well as the $D(\bar{D})$ mesons are assumed to be of harmonic oscillator, with strengths $\beta$ and $\beta_{D}$ respectively. As has already been mentioned in the previous section, the value of the parameter $\beta$ is fitted from the rms radius of the specific charmonium state. The strength of the wave function for the $D(\bar{D})$ as well as the strength for the decay of the charmonium state to $D(\bar{D})$ are fitted from the decay of $\psi(3770)$ to $D(\bar{D})$ in vacuum. As one might observe from the expressions for the decay widths of the charmonium states to $D(\bar{D})$ given by equations (30), (31) and (32), the dependence of these decay widths on the medium is through the magnitude of the center of mass momentum of the produced $D(\bar{D})$ meson, $p_{D}$, written as $\mathrm{x}$ in dimensionless units $\left(x=p_{D} / \beta_{D}\right)$. The value of $p_{D}$ depends on the medium through the modifications of the masses of the charmonium state, as well as on the masses of the $D \equiv\left(D^{0}, D^{+}\right)$and $\bar{D} \equiv\left(\bar{D}^{0}, D^{-}\right)$mesons, as can be seen from its expression given by (29).

In Figs. 9 and 10, we show the partial decay widths of (i) $\psi \rightarrow D^{+} D^{-}$, (ii) $\psi \rightarrow D^{0} \bar{D}^{0}$ and (iii) $\psi \rightarrow D^{+} D^{-}$ and $\psi \rightarrow D^{0} \bar{D}^{0}$, for the charmonium state, $\psi$ as $J / \psi, \psi(3686)$ and $\psi(3770)$ for zero temperature, for the symmetric $(\eta=0)$ and asymmetric (with $\eta=0.5)$ hadronic matter. These have been shown for the value of strangeness fraction, $f_{s}=0.5$ and have been compared with the case of nuclear matter $\left(f_{s}=0\right)$. For the case of symmetric nuclear matter $\left(\eta=0\right.$ and $\left.f_{s}=0\right)$, the decay width of the charmonium state $\psi(3686)$ vanishes below a density of $\rho_{B}=0.6 \rho_{0}$ as the mass of the charmonium state is smaller than the mass of the $D(\bar{D})$ pair $\left(D^{+} D^{-}\right.$as well as $\left.D^{0} \bar{D}^{0}\right)$ for these densities. For densities above this value of the density, the magnitude of $p_{D}$ is seen to increase with density. However, at high densities, the increase is seen to be much slower when the density is increased still further. The polynomial part $\left(1+\frac{2 r^{2}\left(1+r^{2}\right)}{\left(1+2 r^{2}\right)\left(3+2 r^{2}\right)\left(1-3 r^{2}\right)} x^{2}\right)^{2}$, in the expression for the decay width of $\psi(3686)$ given by equation (31) remains unity for densities less than $0.6 \rho_{0}$ (since $x=p_{D} / \beta_{D}$ vanishes for these densities) and is seen to decrease with density, for higher densities. The decay width for the decay of $\psi(3686) \rightarrow D \bar{D}$, which is a combined effect of the polynomial as well as the gaussian parts, is seen to have an initial increase with density and then a drop as the density is further increased. However, one does not observe any nodes in the partial width even up to a density of about $6 \rho_{0}$. With the inclusion of strangeness in the medium, the behavior of the decay width of $\psi(3686) \rightarrow D \bar{D}$ with density is seen to be similar to as in the nuclear matter. However, due to smaller values of the masses of the $D(\bar{D})$ mesons, leading to higher values of the center of mass momentum, $p_{D}$, there is faster suppression of the polynomial part of the decay width leading to nodes in the decay width of $\psi(3686)$ to $D^{+} D^{-}$as well as to $D^{0} \bar{D}^{0}$ at a density of about $4.5 \rho_{0}$, as can be seen in the subplot (c) in figure 9 Such nodes have been already observed in the literature in the decay widths of the charmonium states $\psi(3686)$ and $\psi(3770)$ to $D \bar{D}$ [57], when the drop in the masses of the $D(\bar{D})$ mesons was incorporated. The decay of $\psi(3770) \rightarrow D \bar{D}$ is possible in vacuum as the mass of $\psi(3770)$ is higher than the mass of the $D \bar{D}$ pair. For $\psi(3770)$, there is seen to be initial increase in $p_{D}$ as a function of density and then a drop with further increase in the density when the modifications of the $D(\bar{D})$ masses as computed in the present investigation, are taken into account. However, there is seen to a strong suppression of the decay width with density due to the polynomial part, $\left(1-\frac{1+r^{2}}{5\left(1+2 r^{2}\right)} x^{2}\right)^{2}$ of the expression for the decay width given in equation (32). Consequently, in the present investigation, there are seen to be nodes in the partial decay widths of the charmonium states $\psi(3686)$ and $\psi(3770)$ to $D \bar{D}$ pairs at densities of about $4.5 \rho_{0}$ and $2.8 \rho_{0}$ respectively (see subplots (c) and (e) of figure 9) in symmetric strange hadronic matter, when the mass modifications of the $D$ and $\bar{D}$ mesons are taken into account, but the modifications of the charmonium masses are neglected. One observes the nodes in the decay widths for these charmonium states also for the asymmetric hadronic matter, as can be seen from subplots of (c) and (e) of figure 10. However, the nodes in the decay widths are observed at smaller values as compared to the isospin symmetric case, since the value of $p_{D}$ increases with isospin asymmetry in the medium, due to larger drop in the $D(\bar{D})$ masses. On the other hand, there are seen to be significant modifications to the partial decay widths of $\psi(3686)$ and $\psi(3770)$, when the mass modifications of these charmonium states are also taken into account, as can be seen from the subplots (d) and (f) in figures 9 and 10. This is due to the fact that the value of $p_{D}$ decreases for the latter case, due to the drop in the charmonium masses in the medium, as can be seen from the expression for $p_{D}$ given by equation (29). The behavior of the decay widths of an initial rise and then a drop as we increase the density, is still observed, when the mass modifications of the charmonium states as well as $D$ and $\bar{D}$ are taken into account. However, the suppression of the decay width is not as large as in the case of neglecting the charmonium mass (due to the smaller value of $p_{D}$ for a given density) and consequently, no nodes are observed even up to a density of about $6 \rho_{0}$. For the case of $\eta=0.5$, as can be seen from the subplot (f) of figure 10, the decay of the state $\psi(3770)$ to $D^{+} D^{-}$is a possibility for all densities, whereas its decay to $D^{0} D^{0}$ does not become possible above a density of about $2.8 \rho_{0}$ for $f_{s}=0$, as can also be seen from subplots (a) and (b) of figure 7 For $J / \psi$, for densities above the threshold density for the decay of $J / \psi$ to $D \bar{D}$ pairs, the decay width is seen to increase monotonically with density, and there is no polynomial part in the the expression 
of the decay width of $J / \psi$ (see equation (30) ) and so no nodes are observed in the decay width of $J / \psi$ to $D \bar{D}$ pair.

\section{SUMMARY}

To summarize, in the present investigation, we have studied the in-medium properties of $D$ and $\bar{D}$ mesons in isospin asymmetric strange hadronic matter at finite temperatures by generalizing the chiral SU(3) model to SU(4). The properties of $D$ mesons are modified due to interaction with the baryons (nucleons and hyperons) and also with the scalar fields $\sigma, \zeta, \delta$ and the dilaton field $\chi$. The scalar meson-baryon coupling parameters of the model are fitted from the vacuum hadron masses and the parameters $d_{1}$ and $d_{2}$ occurring in the range terms are fitted from the low energy $K N$ scattering data. We observe that for a given value of density and isospin asymmetry, the strangeness of the medium is seen to lead to decrease in the masses of $D$ and $\bar{D}$ mesons and the masses of the $\bar{D}$ mesons are seen to be more sensitive to the strangeness of the medium as compared to the masses of the $D$ mesons. On the other hand, in the isospin asymmetric strange hadronic matter, the masses of the $D$ mesons are observed to depend more strongly on the isospin asymmetry of the medium, as compared to the masses of the $\bar{D}$ mesons. The mass shifts for the $D$ mesons obtained in the present calculations are observed to be appreciable and the values are in agreement with the calculations of the mass shifts in the QMC model [27] as well as in QCD sum rule approach [29, 30]. On the other hand, the calculations with the coupled channel approach [32, 34] observed only small mass modifications of the $D$ and $\bar{D}$ mesons in the medium. The $D$ mesons are also observed to have attractive mass shifts within the calculations using a coupled channel approach based on heavy quark symmetry [48]. However, the mass shifts in Ref. [48] are observed to be much smaller than what is observed in the present work. The attractive mass shifts of the $D$ mesons can give rise to the possibility of the $D$-mesic nuclei, which have been investigated in Ref. [48]. The ratios $D^{+} / D^{0}$ and $D^{-} / \overline{D^{0}}$ of their production cross sections as well as collective flow, could be promising observables to study the effect of strangeness fraction of the medium on the properties of $D$ and $\bar{D}$ mesons. The isospin dependence of $D^{+}$and $D^{0}$ masses is seen to be a dominant medium effect at high densities, whereas, for the $D^{-}$and $\bar{D}^{0}$, one sees that, even though these have a strong density dependence, their in-medium masses remain similar at a given value for the isospin-asymmetry parameter $\eta$. As the production of the $D$ and $\bar{D}$ mesons are dominantly by the decay of the charmonium states to $D^{+} D^{-}\left(D^{0} \bar{D}^{0}\right)$ pairs, the production cross-sections for the ratios of $D^{+} / D^{0}$ as well as $D^{-} / \bar{D}^{0}$ are expected to be modified substantially in the medium due to the stronger isospin dependence of the $D$ meson doublet as compared to the $\bar{D}$ meson doublet, whereas, the parameter, $v_{2}$ for the anisotropic collective flow, is expected to modify the ratio of $D^{+} / D^{0}$ more appreciably as compared to the flow of the ratio $D^{-} / \overline{D^{0}}$. However, due to the presence of the light antiquark (quark) in the $D(\bar{D})$, they might undergo substantial decay in the hadronic environment, which could make it difficult to extract clean signals for the medium modifications of the $D$ and $\bar{D}$ mesons in the isospin asymmetric strange hadronic medium, from the experimental data arising from isospin asymmetric heavy ion collision experiments.

We have also investigated in the present work, the effects of density, temperature, isospin asymmetry and strangeness fraction of the strange hadronic medium on the masses of the charmonium states $J / \psi, \psi(3686)$ and $\psi(3770)$, arising due to modification of the scalar dilaton field, $\chi$, which simulates the gluon condensates of QCD, within the chiral $\mathrm{SU}(3)$ model, as well as due to the modifications of the scalar fields $\sigma$ and $\zeta$, when we account for the quark mass term in the trace of the energy momentum tensor in QCD. We have taken into consideration the finite quark masses through the explicit chiral symmetry breaking term in the chiral effective model, for estimating the medium modification of the gluon condensates, from which we obtain the mass modifications of the charmonium states in the present investigation. Due to the modifications of the charmonium wave functions in the hadronic medium, the radii of the charmonium states are modified and these have been computed in the present work. For the charmonium states $\psi(3686)$ and $\psi(3770)$, there is seen to be increase in their root mean square radii, with density, arising due to the decrease in the strengths of their harmonic oscillator wave functions in the hadronic medium. The change in these wave functions are computed from the mass shifts of these charmonium states as calculated in the present investigation.

The partial decay widths for the decay of the charmonium states to $D^{+} D^{-}$as well as $D^{0} \bar{D}^{0}$ have also been investigated in the hadronic medium, which are seen to have nodes for the charmonium states $\psi(3686)$ and $\psi(3770)$, as has already been observed in the literature [57], when we account for the mass modifications of the $\mathrm{D}$ and $\bar{D}$ mesons, but do not consider the in-medium masses of the charmonium states. However, while accounting for the mass modifications of the charmonium states as well, the partial decay widths are seen to be modified significantly, and there are no nodes in the partial decay widths observed up to a density of about $6 \rho_{0}$. The change in the mass of $J / \psi$ with the density is observed to be small at nuclear matter saturation density and is in agreement with the QCD sum rule calculations. There is seen to be appreciable drop in the in-medium masses of excited charmonium states $\psi(3686)$ and $\psi(3770)$ with density. The mass drop of the excited charmonium states $\psi(3686)$ and $\psi(3770)$ are large enough 
to be seen in the dilepton spectra emitted from their decays in experiments involving $\bar{p}$-A annihilation in the future facility at GSI, provided these states decay inside the nucleus. The in-medium properties of the excited charmonium states $\psi(3686)$ and $\psi(3770)$ can be studied in the dilepton spectra in $\bar{p}$-A experiments in the future facility of the FAIR, GSI [25]. The mass shift of the charmonium states in the hot hadronic medium seem to be appreciable at high densities as compared to the temperature effects on these masses, and these should show in observables like the production of these charmonium states in the compressed baryonic matter experiment at the future facility at GSI, where baryonic matter at high densities and moderate temperatures will be produced.

The medium modifications of the $D$ meson masses can lead to a suppression in the $J / \psi$ yield in heavy-ion collisions, since the excited states of the $J / \psi$, as well as $J / \psi$, can decay to $D \bar{D}$ pairs in the dense hadronic medium. The medium modifications of the masses of the charmonium states as well as the $D$ and $\bar{D}$ mesons have been considered in the present investigation in strange hadronic medium at finite temperatures. In the present investigation, the charmonium masses have been calculated taking into account the finite quark mass term in the trace of the energy momentum tensor and the mass drop for the charmonium state is seen to be appreciably smaller than when this is not taken into account. We observe that the excited charmonium states, $\psi(3686)$ as well as $\psi(3770)$ have a possibility of decaying to $D \bar{D}$ pairs, whereas $J / \psi$ is observed to decay to $D \bar{D}$ pair only above certain value of the density depending on the isospin asymmetry and temperature of the medium. The conclusions for the possibility of the decay of the charmonium states to the $D \bar{D}$ pairs are found to be substantially modified when the in-medium masses of the charmonium states are calculated from the gluon condensate in the medium, obtained by neglecting the quark mass term in the energy momentum tensor in QCD. The strong density, strangeness and isospin dependence of the $D(\bar{D})$ meson masses, charmonium masses as well as the partial decay widths of the charmonium states decaying to $D \bar{D}$ pairs, in hot isospin asymmetric strange hadronic matter can be tested in the asymmetric heavy-ion collision experiments at future GSI facility [25].

We thank Arshdeep Singh Mehta for discussions. Financial support from Department of Science and Technology, Government of India (project no. SR/S2/HEP-21/2006) is gratefully acknowledged. One of the authors (AM) is grateful to the Frankfurt Institute for Advanced Research (FIAS), University of Frankfurt, for warm hospitality and acknowledges financial support from Alexander von Humboldt Stiftung when this work was initiated.

[1] G. Agakichiev et al. (CERES collaboration), Phys. Rev. Lett. 75, 1272 (1995); G. Agakichiev et al. (CERES collaboration), Phys. Lett. B 422, 405 (1998); G. Agakichiev et al. (CERES collaboration), Nucl. Phys. A661, 23c (1999).

[2] N. Masera et al. (HELIOS-3 collaboration), Nucl. Phys. A590, 93c (1995).

[3] R. J. Porter et al. (DLS collaboration), Phys. Rev. Lett. 79, 1229 (1997); W. K. Wilson et al. (DLS collaboration), Phys. Rev. C 57, 1865 (1998).

[4] E. L. Bratkovskaya and W. Cassing, Nucl. Phys. A619, 413 (1997).

[5] W. Cassing and E. L. Bratkovskaya, Phys. Rep. 308, 65 (1999).

[6] A. Mishra, J. C. Parikh and W. Greiner, J. Phys. G 28, 151 (2002).

[7] A. Mishra, J. Reinhardt, H. Stöcker and W. Greiner, Phys. Rev. C 66, 064902 (2002).

[8] G. Q. Li, C. M. Ko and G. E. Brown, Nucl. Phys. A606, 568 (1996).

[9] D. K. Srivastava, B. Sinha, and Charles Gale, Phys. Rev. C 53, R567 (1996).

[10] D. K. Srivastava, Jour. Phys. G : Nucl. Part. Phys. 35, 104026 (2008); D. K. Srivastava and Rupa Chatterjee, Phys. Rev. C 80, 054914 (2009).

[11] Jitesh R. Bhatt and V. Sreekanth, Int. Jour. Mod. Phys. E 19, 299 (2010).

[12] F. Laue et al., Phys. Rev. Lett. 82, 1640 (1999).

[13] C. Sturm et al., Phys. Rev. Lett. 86, 39 (2001).

[14] A. Förster et al., KaoS Collaboration, J. Phys. G 28, 2011 (2002).

[15] M. Menzel et al., Phys. Lett. B 495, 26 (2000).

[16] C. M. Ko, J. Phys. G 27, 327 (2001).

[17] G. Q. Li, C.-H. Lee and G. E. Brown, Nucl. Phys. A625, 372 (1997).

[18] S. Pal, C. M. Ko, and Z.-W. Lin, Phys. Rev. C 64, 042201 (2001).

[19] W. Cassing, L. Tolos, E. L. Bratkovskaya and A. Ramos, Nucl. Phys. A727, 59 (2003).

[20] A. Mishra, E. L. Bratkovskaya, J. Schaffner-Bielich, S. Schramm and H. Stöcker, Phys. Rev. C 70, 044904 (2004).

[21] A. Mishra and S. Schramm, Phys. Rev. C 74, 064904 (2006), A. Mishra, S. Schramm and W. Greiner, Phys. Rev. C 78, 024901 (2008).

[22] Amruta Mishra, Arvind Kumar, Sambuddha Sanyal, S. Schramm, Eur. Phys, J. A 41, 205 (2009).

[23] D.B. Kaplan and A.E. Nelson, Phys. Lett. B 175, 57 (1986).

[24] http://www.jlab.org/ 
[25] see e.g. http://www.gsi.de/fair/experiments/CBM/

[26] http://www-panda.gsi.de/

[27] K. Tsushima, D. H. Lu, A. W. Thomas, K. Saito, and R. H. Landau, Phys. Rev. C 59, 2824 (1999); A. Sibirtsev, K. Tsushima, and A. W. Thomas, Eur. Phys. J. A 6, 351 (1999).

[28] A. Hayashigaki, Phys. Lett. B 487, 96 (2000).

[29] T. Hilger, R. Thomas, B. Kämpfer, Phys. Rev. C 79, 025202 (2009).

[30] P. Morath, W. Weise and S. H. Lee, 17th Autumn school: Lisbon 1999, QCD: Perturbative or Nonperturbative? Singapore: World Scientific, p. 425.

[31] L.Tolos, J. Schaffner-Bielich and A. Mishra, Phys. Rev. C 70, 025203 (2004).

[32] L. Tolos, J. Schaffner-Bielich and H. Stöcker, Phys. Lett. B 635, 85 (2006).

[33] T. Mizutani and A. Ramos, Phys. Rev. C 74, 065201 (2006).

[34] L.Tolos, A. Ramos and T. Mizutani, Phys. Rev. C 77, 015207 (2008).

[35] E.A. Veit, B. K. Jennings, A. W. Thomas, R. C. Berrett, Phys. Rev. D 31, 1033 (1985); L. Tolos, A. Ramos, A. Polls and T.T.S. Kuo, Nucl. Phys. A 690, 547 (2001); V. Koch, Phys. Lett. B 337, 7 (1994); P. B. Siegel and W. Weise, Phys. Rev. C 38, 2221 (1998).

[36] M. Arutso et al. (CLEO Collaboration), Phys. Rev. Lett. 86, 4479 (2001).

[37] R. Mizuk et al. (Belle Collaboration), Phys. Rev. Lett. 94, 122002 (2005).

[38] R. Chistov et al. (BELLE Collaboration), Phys. Rev. Lett. 97, 162001 (2006).

[39] B. Aubert et al. (BABAR Collaboration), Phys. Rev. Lett. 97, 232001 (2006).

[40] B. Aubert et al. (BABAR Collaboration), Phys. Rev. Lett. 98, 012001 (2007).

[41] R. Mizuk et al. (Belle Collaboration), Phys. Rev. Lett. 98, 262001 (2007).

[42] L. Tolos, C. García-Recio, and J. Nieves, Phys. Rev. C 80, 065202 (2009).

[43] J. Hofmann and M.F.M.Lutz, Nucl. Phys. A 763, 90 (2005).

[44] K. Kawarabayashi and M. Suzuki, Phys. Rev. Lett. 16, 255 (1966); Riazuddin and Fayyazuddin, Phys. Rev. 147, 1071 (1966).

[45] M.F.M. Lutz and C.L. Korpa, Phys. Lett. B 633, 43 (2006).

[46] C. Garcia-Recio, V. K. Magas, T. Mizutani, J. Nieves, A. Ramos, L. L. Salcedo and L. Tolos, Phys. Rev. D 79, 054004 (2009)

[47] D. Gamermann, C. Garcia-Recio, J. Nieves, L. L. Salcedo and L. Tolos, Phys. Rev. D 81, 094016 (2010).

[48] C. Garcia-Recio, J. Nieves and L. Tolos, Phys. Lett. B 690, 369 (2010).

[49] Laura Tolos, Raquel Molina, Daniel Gamermann and Eulogio Oset, Nucl. Phys. A 827, 249c (2009).

[50] M. Gonin et al., NA50 Collaboration, Nucl. Phys. A610, 404c (1996).

[51] M. C. Abreu et al., NA50 Collaboration, Eur. Phys. J. C 14, 443 (200).

[52] L. Rammello et al., NA50 Collaboration, Nucl. Phys. A638, 261c (1998); M. C. Abreu et al., Phys. Lett. B 450, 456 (1999); L. Ramello et al., Nucl. Phys. A715, 242 (2003).

[53] L. Antoniazzi et al., E705 Collaboration, Phys. Rev. Lett. 70, 383 (1993); Y. Lemoigne et al., Phys. Lett. B 113, 509 (1982).

[54] Ye. S. Golubeva, E. L. Bratkovskaya, W. Cassing, and L. A. Kondratyuk, Eur. Phys. J. A 17, 275 (2003).

[55] S. H. Lee and C. M. Ko, Phys. Rev. C 67, 038202 (2003).

[56] A. Hayashigaki, Prog. Theor. Phys. 101, 923 (1999).

[57] B. Friman, S. H. Lee and T. Song, Phys. Lett. B 548, 153 (2002).

[58] G. Krein, A. W. Thomas, K. Tsushima, Phys. Lett. B 697, 136 (2011).

[59] T. Matsui and H. Satz, Phys. Lett. B 178, 416 (1986).

[60] J. P. Blaizot and J. Y. Ollitrault, Phys. Rev. Lett. 77, 1703 (1996).

[61] A. Sibirtsev, K. Tsushima, K. Saito and A.W. Thomas, Phys. Lett. B484, 25 (2000).

[62] A. Capella and E. G. Ferreiro, Eur. Phys. J. C 42, 419 (2005); N. Armesto, A. Capella, E. G. Ferreiro, A. Kaidalov and D. Sousa, Nucl. Phys. A698, 583c (2002).

[63] Sean Gavin and Ramona Vogt, Nucl. Phys. A610, 442c (1996); Sean Gavin and Ramona Vogt, Phys. Rev. Lett. 78, 1006 (1997); S. Gavin and R. Vogt, Nucl. Phys. B345, 104 (1990).

[64] B. Zhang, C. M. Ko, B. A. Li, Z. Lin, and B. H. Sa, Phys. Rev. C 62, 054905 (2000).

[65] W. Cassing and E. L. Bratkovskaya, Nucl. Phys. A623, 570 (1997).

[66] E. L. Bratkovskaya, W. Cassing and H. Stöcker, Phys. Rev. C 67, 054905 (2003).

[67] W. Cassing and C.M. Ko, Phys. Lett. B396, 39 (1997); W. Cassing, E.L. Bratkovskaya, S. Juchem, Nucl. Phys. A674, 249 (2000).

[68] P. Papazoglou, D. Zschiesche, S. Schramm, J. Schaffner-Bielich, H. Stöcker, and W. Greiner, Phys. Rev. C 59, 411 (1999).

[69] W. Liu, C. M. Ko, and Z. W. Lin, Phys. Rev. C 65, 015203 (2001).

[70] Arvind Kumar and Amruta Mishra, Phys, Rev. C 81, 065204 (2010).

[71] D. Zschiesche, A. Mishra, S. Schramm, H. Stöcker and W. Greiner, Phys. Rev. C 70, 045202 (2004).

[72] A. Mishra, K. Balazs, D. Zschiesche, S. Schramm, H. Stöcker, and W. Greiner, Phys. Rev. C 69, 024903 (2004).

[73] A. Mishra, E. L. Bratkovskaya, J. Schaffner-Bielich, S.Schramm and H. Stöcker, Phys. Rev. C 69, 015202 (2004).

[74] Amruta Mishra and Arindam Mazumdar, Phys. Rev. C 79, 024908 (2009). 
[75] Arvind Kumar and Amruta Mishra, Phys. Rev. C 82, 045207 (2010).

[76] S.Weinberg, Phys. Rev. 166, 1568 (1968).

[77] S. Coleman, J. Wess, B. Zumino, Phys. Rev. 177, 2239 (1969); C.G. Callan, S. Coleman, J. Wess, B. Zumino, Phys. Rev. 177, 2247 (1969).

[78] W. A. Bardeen and B. W. Lee, Phys. Rev. 177, 2389 (1969).

[79] J. Schechter, Phys. Rev. D 21, 3393 (1980).

[80] Thomas D. Cohen, R. J. Furnstahl and David K. Griegel, Phys. Rev. C 45, 1881 (1992).

[81] B.Borasoy and U-G. Meissner, Int. J. Mod. Phys. A 11, 5183 (1996).

[82] D. Röder, J. Ruppert, and D.H. Rischke, Phys. Rev. D 68, 016003 (2003).

[83] F. Klingl etal., Phys. Rev. Lett. 82, 3396 (1999).

[84] M.E. Peskin, Nucl. Phys. B156, 365 (1979).

[85] A. Le Yaouanc, L. Oliver, O. Pene, J. C. Raynal, Phys. Rev. D 8, 2223 (1973); ibid, Phys. Rev. D 9, 1415 (1974); ibid, Phys. Rev. D 11, 1272 (1975).

[86] T. Barnes, F. E. Close, P. R. Page and E. S. Swanson, Phys. Rev. D 55, 4157 (1997).

[87] A Le Yaouanc et al, Phys. Lett. B 71, 397 (1977).

[88] K. Nakamura et al (Particle Data Group) J. Phys. G 37, 075021 (2010).

[89] Su Houng Lee and Kenji Morita, Phys. Rev. D 79, 011501(R) (2009).

[90] Sugsik Kim, Su Houng Lee, Nucl. Phys. A 679, 517 (2001).

[91] Cesareo A. Dominguez, Marcelo Loewe, J. Cristobal Rojas and Yingwen Zhang arXiv 0908.2709 [hep-ph].

[92] Kenji Morita and Su Houng Lee, Phys. Rev. Lett. 100, 022301 (2008). 